\title{
An inclination lemma for normally hyperbolic manifolds with an application to diffusion
}

\author{
Lara Sabbagh * \\ Mathematics Institute, University of Warwick, email: l.el-sabbagh@warwick.ac.uk
}

September 25, 2018

\begin{abstract}
Let $(M, \Omega)$ be a smooth symplectic manifold and $f: M \rightarrow M$ be a symplectic diffeomorphism of class $C^{l}(l \geq 3)$. Let $N$ be a compact submanifold of $M$ which is boundaryless and normally hyperbolic for $f$. We suppose that $N$ is controllable and that its stable and unstable bundles are trivial. We consider a $C^{1}$-submanifold $\Delta$ of $M$ whose dimension is equal to the dimension of a fiber of the unstable bundle of $T_{N} M$. We suppose that $\Delta$ transversely intersects the stable manifold of $N$. Then, we prove that for all $\varepsilon>0$, and for $n \in \mathbb{N}$ large enough, there exists $x_{n} \in N$ such that $f^{n}(\Delta)$ is $\varepsilon$-close, in the $C^{1}$ topology, to the strongly unstable manifold of $x_{n}$.

As an application of this $\lambda$-lemma, we prove the existence of shadowing orbits for a finite family of invariant minimal sets (for which we do not assume any regularity) contained in a normally hyperbolic manifold and having heteroclinic connections. As a particular case, we recover classical results on the existence of diffusion orbits (Arnold's example).
\end{abstract}

\section{Introduction}

In his famous note Arn64, Arnold gave the first example of a three-degree-of-freedom system where diffusion orbits shadowing whiskered tori were constructed. More precisely, the system admits orbits for which the action undergoes a drift of length independent of the size of the perturbation. Arnold's example was chosen so that the Lagrangian invariant tori in the unperturbed system break down under the perturbation and give rise to partially hyperbolic tori in the perturbed system.

The diffusion mechanism is then based on the existence of a transition chain, that is, a family of invariant minimal tori with heteroclinic connections. One gets the orbits shadowing the extremal tori of this chain by an "obstruction argument" satisfied by each torus of the chain. This obstruction argument was first proved in the paper [Mar96] as a corollary of a partially hyperbolic $\lambda$-lemma. The proof was then improved in [FM00] (see also [Cre00]).

In the present paper, we prove a $\lambda$-lemma (also called inclination lemma) for normally hyperbolic invariant manifolds, which turns out to be a new tool for proving the obstruction argument as well as several generalizations. This $\lambda$-lemma deals with normally hyperbolic manifolds instead of partially hypebolic tori. This is not a genuine restriction since one can in general embed

\footnotetext{
${ }^{*}$ This work was partially supported by the EPSRC grant EP/J003948/1.
} 
partially hyperbolic tori into their central manifolds which, as a rule, are normally hyperbolic. In that respect, this paper generalizes the results of [Mar96], Cre00] and [FM00], and enables us to significatively simplify the previous proofs. Moreover, our $\lambda$-lemma can be applied to more general systems than that of Arnold ([DDLLS06], [DH11, GR07, GR09, ,...) and can relate to the variational methods (which is another approach to diffusion problems) where significant contributions were given by Bernard, Bessi, Cheng, Kaloshin and many others.

We first state and prove a $\lambda$-lemma for normally hyperbolic invariant manifolds. Given a normally hyperbolic invariant manifold $N$ for a diffeomorphism $f$, we consider a submanifold that transversely intersects the stable manifold of $N$ and whose dimension is equal to the dimension of a fiber of the unstable bundle. We prove that under iteration by $f$, this submanifold is as close as desired (in the $C^{1}$ topology) to a suitable unstable leaf. The $\lambda$-lemma will enable us to prove the existence of drifting orbits along a chain of invariant minimal sets contained in a normally hyperbolic manifold, without any assumption on the nature of the invariant sets (in particular, they do not need to be submanifolds). As an easy particular case, we recover Arnold's example. In addition, the $\lambda$-lemma applies immediately to the examples of Delshams, De La Llave and Seara (see [DDLLS06] and the references therein) and yields the diffusion orbits.

In this paper, we will limit ourselves to the symplectic case and we will assume that our normally hyperbolic manifold has trivial stable and unstable bundles (this will in particular give us easy regularity conditions for the lamination of the invariant manifolds). This will be no restriction to us since all the applications that we have in mind will fall into this category (diffusion orbits, Easton's windows,...). Moreover, we will adopt a very basic point of view and depict the geometry of the iterates of our transverse manifolds instead of using a more synthetic method (fixed point theorem for instance). In particular, this will enable us to directly use our various computations for the construction of windows and for estimating the transition times in a subsequent work. As a counterpart, we will have to use the existence of "controlled" straightening neighborhoods for our manifold, which requires the previous (maybe unnecessary) assumptions.

The normally hyperbolic invariant manifolds we consider will be compact for technical simplicity but the non-compactness could easily be replaced with uniform lower bounds for the first and second derivatives of our diffeomorphisms and the constants of hyperbolicity (see (11) below). Finally, let us point out that eventhough we prove the $\lambda$-lemma for discrete systems, as usual analogous results hold for the continuous time Hamiltonian systems.

Acknowledgments. I would like to thank Jean-Pierre Marco for having suggested these questions to me and for having generously shared his ideas with me.

\section{A reminder on normally hyperbolic invariant manifolds and convention}

We begin with a reminder on normally hyperbolic manifolds in a general context and then specialize to the symplectic case where we can use a "controlled" straightening neighborhood in which it is easy to depict the geometry of the invariant foliations induced by normal hyperbolicity.

\subsection{General definitions}

Let $M$ be a smooth $n$-dimensional manifold $(n \geq 3)$ and $f: M \rightarrow M$ be a $C^{l}$-diffeomorphism $(l \geq 1)$ which leaves a smooth boundaryless compact submanifold $N$ of $M$ invariant. Given a Riemannian metric $\|\cdot\|$ on $M$ and a subbundle $E$ of $T_{N} M$ invariant under $D f$, we set:

$$
\operatorname{norm}\left(D f_{\left.\right|_{E}}\right)=\sup \left\{\left\|D f(a)_{\left.\right|_{E_{a}}}\right\| ; a \in N\right\}, \quad \operatorname{conorm}\left(D f_{\left.\right|_{E}}\right)=\left(\operatorname{norm}\left(D f_{\left.\right|_{E}}^{-1}\right)\right)^{-1} \text {. }
$$


Definition 2.1. Let $q \leq l\left(q \in \mathbb{N}^{*}\right)$. The manifold $N$ is q-normally hyperbolic for $f$ if the tangent bundle of $M$ restricted to $N$ splits into three continuous subbundles $T_{N} M=T N \oplus E^{s} \oplus E^{u}$ invariant under $D f$, such that

$$
\operatorname{norm}\left(D f_{\left.\right|_{E} s}\right)<\left(\operatorname{conorm}\left(D f_{\left.\right|_{T N}}\right)\right)^{q} \leq 1 \leq\left(\operatorname{norm}\left(D f_{\left.\right|_{T N}}\right)\right)^{q}<\operatorname{conorm}\left(D f_{\left.\right|_{E} u}\right) .
$$

This says that the behavior of $f$ normal to $N$ dominates the tangent behavior of $f^{q}$ and is hyperbolic.

Now we state the local stable/unstable manifolds theorem. We do not mean to give the most general possible results, we rather limit ourselves to those which are strictly necessary for our purposes. For a more elaborate study on invariant manifolds, we refer to [HPS77, Cha04] and BB].

Theorem [HPS77]. Let $f, M$ and $N$ be as above. We suppose that $N$ is q-normally hyperbolic for $f$. Then if $d$ is the distance associated with the Riemannian metric on $M$, the following properties hold true:

1. Existence, characterization and smoothness. There exists a neighborhood $\mathcal{O}$ of $N$ in $M$ such that the sets:

$$
W_{l o c}^{s}(N)=\left\{y \in \mathcal{O} ; f^{n}(y) \in \mathcal{O}, \forall n \in \mathbb{N}\right\} \text { and } W_{l o c}^{u}(N)=\left\{y \in \mathcal{O} ; f^{-n}(y) \in \mathcal{O}, \forall n \in \mathbb{N}\right\}
$$

are $C^{q}$-manifolds that satisfy

- $\left.\forall y \in W_{l o c}^{s}(N), \forall \rho \in\right] \operatorname{norm}\left(D f_{\left.\right|_{E}}\right) ; \operatorname{conorm}\left(D f_{\left.\right|_{T N}}\right)\left[, \lim _{n \rightarrow \infty} \rho^{-n} d\left(f^{n}(y), N\right)=0\right.$,

- $\left.\forall y \in W_{l o c}^{u}(N), \forall \rho \in\right] \operatorname{norm}\left(D f_{\left.\right|_{T N}}\right) ; \operatorname{conorm}\left(D f_{\left.\right|_{E} u}\right)\left[, \lim _{n \rightarrow \infty} \rho^{n} d\left(f^{-n}(y), N\right)=0\right.$.

Moreover, $W_{\text {loc }}^{u}(N)$ and $W_{l o c}^{s}(N)$ are tangent to $T N \oplus E^{u}$ and $T N \oplus E^{s}$ respectively at each point of $N$.

2. Lamination. There exist two f-invariant laminations of $W_{l o c}^{u}(N)$ and $W_{l o c}^{s}(N)$, the leaves of which are unstable and stable leaves $W_{l o c}^{u u}(x)$ and $W_{l o c}^{s s}(x)$ associated with the points of $N$, defined as follows:

$$
\begin{aligned}
& W_{l o c}^{s s}(x)=\left\{y \in \mathcal{O} ; \lim _{n \rightarrow \infty} d\left(f^{n}(y), f^{n}(x)\right)=0\right\} \text { and } \\
& W_{l o c}^{u u}(x)=\left\{y \in \mathcal{O} ; \lim _{n \rightarrow \infty} d\left(f^{-n}(y), f^{-n}(x)\right)=0\right\} .
\end{aligned}
$$

These leaves are $C^{q}$ and tangent to the fibers $E_{x}^{u}$ and $E_{x}^{s}$ at each point $x$ of $N$.

Note that one gets the global stable (resp. unstable) manifolds by taking the union of the inverse (resp. direct) images of the local ones as follows:

$$
W^{s}(N)=\bigcup_{n \in \mathbb{N}} f^{-n}\left(W_{l o c}^{s}(N)\right) \text { and } W^{u}(N)=\bigcup_{n \in \mathbb{N}} f^{n}\left(W_{l o c}^{u}(N)\right) .
$$

The same holds for the leaves:

$$
W^{s s}(x)=\bigcup_{n \in \mathbb{N}} f^{-n}\left(W_{l o c}^{s s}\left(f^{n}(x)\right)\right) \text { and } W^{u u}(x)=\bigcup_{n \in \mathbb{N}} f^{n}\left(W_{l o c}^{u u}\left(f^{-n}(x)\right)\right) .
$$

These are immersed $C^{q}$-submanifolds of $M$. In the rest of the paper, we will drop the subscript $l o c$ from the notation. The local and the global invariant manifolds will be denoted by $W^{s, u}(N)$ since the context will always be clear. The same holds for the global and local leaves. 
Definition 2.2. Let $N$ be a q-normally hyperbolic manifold for $f(q \leq l)$. We say that $N$ is controllable if the following inequalities hold true

$$
\operatorname{norm}\left(D f_{\left.\right|_{E^{s}}}\right) \cdot \operatorname{norm}\left(D f_{\left.\right|_{T N}}\right)<1 \text { and } \operatorname{conorm}\left(D f_{\left.\right|_{T N}}\right) \cdot \operatorname{conorm}\left(D f_{\left.\right|_{E} u}\right)>1 \text {. }
$$

We set $n_{s}:=\operatorname{dim}\left(E^{s}\right), n_{u}:=\operatorname{dim}\left(E^{u}\right)$ and $n_{0}:=\operatorname{dim}(N)$, so that $n_{0}+n_{s}+n_{u}=n$.

\subsection{Symplectic Geometry and normal hyperbolicity}

Under symplecticity assumptions, the stable and unstable leaves are regular with respect to the points in $N$. More precisely, we have the following proposition which will enable us in the next section to introduce a straightening coordinate system in the vicinity of normally hyperbolic manifolds.

Proposition A. [Marco]. Let $(M, \Omega)$ be a smooth symplectic manifold and let $f$ be $a C^{l}$ symplectic diffeomorphism of $M(l \geq 2)$. We suppose that $N$ is a controllable q-normally hyperbolic manifold for $f(q \leq l)$. Then

- $N$ is symplectic,

- $W^{u}(N)$ and $W^{s}(N)$ are coisotropic,

- $n_{s}=n_{u}$

- for all $x \in N, W^{u u}(x)$ and $W^{s s}(x)$ are isotropic and they coincide with the leaves of the characteristic foliations of $W^{u}(N)$ and $W^{s}(N)$.

The proof of this proposition can be found in Mara. Since the leaves of the characteristic foliations coincide with the leaves $W^{u u}(x)$ and $W^{s s}(x)$, the latter are $C^{q-1}$ with respect to $x$. We get then the regularity we need for Proposition B below.

\subsection{Straightening neighborhood and convention}

Under the assumptions of Proposition A, one can find in the vicinity of a normally hyperbolic manifold a neighborhood in which the invariant manifolds and the leaves are straightened, making it easier to depict the behavior of $f$. More precisely, we have the following proposition.

Proposition B. [Tubular neighborhood and straightening]. Let $M, N$ and $f$ be as in Proposition $A$ with $l \geq 3$. Let $p:=n_{s}=n_{u}$. We suppose that $N$ is 3-normally hyperbolic for $f$ and that its stable and unstable bundles are trivial. Then, there exist a neighborhood $U$ of $N$ in $M$ and $a C^{2}$-diffeomorphism $\varphi: U \longrightarrow V:=N \times B^{p} \times B^{p}$, where $B^{p}$ is an open ball centered at 0 in $\mathbb{R}^{p}$, such that for all $x \in N$ :

1. $\varphi(x)=(x, 0,0)$,

2. $\widetilde{W^{s}}(N):=\varphi\left(W^{s}(N) \cap U\right)=\{(x, s, u) \in V ; u=0\}$,

3. $\widetilde{W}^{u}(N):=\varphi\left(W^{u}(N) \cap U\right)=\{(x, s, u) \in V ; s=0\}$,

4. $\widetilde{W}^{s s}(x):=\varphi\left(W^{s s}(x) \cap U\right)=\left\{(x, s, 0) ; s \in B^{p}\right\}$,

5. $\widetilde{W}^{u u}(x):=\varphi\left(W^{u u}(x) \cap U\right)=\left\{(x, 0, u) ; u \in B^{p}\right\}$. 
The proof is straightforward once Proposition A is known. We will not prove Proposition B, we will content ourselves with the following few remarks. Near $N$, one can always find a tubular neighborhood. The straightening of the invariant manifolds is an immediate consequence of the graph property. We refer to [LMS03] and [HPS77] for details. When $f$ is symplectic, the strongly stable/unstable leaves are straightened the same way.

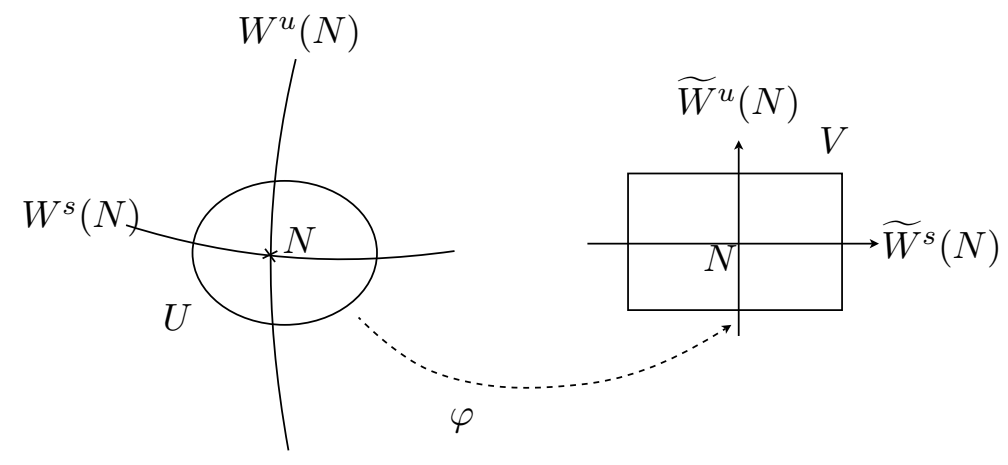

Figure 1: The straightening neighborhood

Convention. The first $B^{p}$ and the second $B^{p}$ in $N \times B^{p} \times B^{p}$ do not play the same role since the first one is the stable direction while the second one is the unstable direction. In order to distinguish them from one another when we want to use them separately, we will add $u$ and $s$ in the notation as follows

$$
N \times B_{s}^{p} \times B_{u}^{p} .
$$

We use the same convention for $N \times \mathbb{R}_{s}^{p} \times \mathbb{R}_{u}^{p}$.

In the rest of the paper, we will identify $N$ with $\varphi(N)=N \times\{0\} \times\{0\}$ for notational symplicity. This will not lead to confusion since the context will always be clear enough.

Let $d$ be the distance associated with the Riemannian metric on $M$. We will equip the neighborhood $V$ defined in Proposition B with the distance given by the sup of $d_{\left.\right|_{N}}$ and the Euclidian distance on $\mathbb{R}^{2 p}$. It is equivalent to the image under $\varphi$ of $d$ since $V$ is relatively compact.

We will use the usual operator norms for the linear applications defined on Banach spaces that we will deal with throughout the paper. We will equip the product spaces with the sup norm and the subsets with the induced norm. For notational simplicity, we will denote all our norms by the same symbol $\|$.$\| ; the context will always be clear enough to avoid ambiguities.$

To prove our results, we will use compositions of linear applications defined on the tangent spaces of some suitable manifolds. They will be normed algebras for the induced norm.

\section{A $\lambda$-lemma for normally hyperbolic manifolds}

In this section, we prove a $\lambda$-lemma for normally hyperbolic manifolds. From now on, we suppose that $f, M, N$ and $\Delta$ are as follows:

- $(M, \Omega)$ is a smooth symplectic Riemannian manifold,

- $f: M \longrightarrow M$ is a symplectic diffeomorphism of class $C^{l}(l \geq 3)$,

- $N$ is a smooth submanifold of $M$, compact and boundaryless, 
- $N$ is a controllable 3-normally hyperbolic manifold for $f$,

- $n_{s}=n_{u}=p$,

- $N$ has trivial stable and unstable bundles,

- $\Delta$ is a $C^{1}$-submanifold of $M$ of dimension $p$ which transversely intersects $W^{s}(N)$ at some point $a$.

We will state two versions of the $\lambda$-lemma. In Section 3.1, we will use the straightening neighborhood given in Section 2.3 to set out a simplified version of the $\lambda$-lemma (Theorem 1) and to properly define the notion of $C^{1}$-convergence. Then, in Section 3.2, we state the $\lambda$ lemma in a more general context (Theorem 2). We devote Sections 4 and 5 to the proofs of these theorems.

\subsection{Theorem 1: in the straightening neighborhood}

In this section, we state the $\lambda$-lemma in the straightening neighborhood. Let us start with fixing the notation. We keep the notation of Proposition B. We will restrict our diffeomorphism $\varphi$ to the open set $\mathcal{U}:=U \cap f^{-1}(U)$, so that $F=\varphi \circ f \circ \varphi^{-1}$ is well defined on $\mathcal{V}:=\varphi(\mathcal{U}) \subset V$ with values in $V$. A point in $V$ will be written as a triple $(x, s, u)$ and $F$ as $\left(F_{x}, F_{s}, F_{u}\right)$ according to the splitting $V=N \times B_{s}^{p} \times B_{u}^{p}$. Up to iterating $\Delta$ if necessary (and resetting the counters), we can suppose that $a \in \mathcal{U}$ without loss of generality, since we are interested in the behavior of $\Delta$ after a large number of iterations.

We introduce the projection $\Pi_{N}: \widetilde{W}^{s}(N) \longrightarrow N$ that sends each $(x, s, 0)$ to $(x, 0,0)$. Let $P:=\varphi(a)=(x, s, 0)$ be the intersection point of $\varphi(\Delta \cap \mathcal{U})$ and $\widetilde{W}^{s}(N)$. We set $P_{0}:=\Pi_{N}(P)$. For $n \geq 1$, we denote by $P^{n}=F^{n}(P)$, and $P_{0}^{n}:=\Pi_{N}\left(P^{n}\right)=F_{\left.\right|_{N}}^{n}\left(P_{0}\right)$ which is the point in $N$ such that $P^{n} \in \widetilde{W}^{s s}\left(P_{0}^{n}\right)$ (see Figure 3). We denote by $\widetilde{\Delta}$ the connected component of $\varphi(\Delta \cap \mathcal{U})$ in $\mathcal{V}$ containing $P$. For all $n \in \mathbb{N}$, we denote by $\widetilde{\Delta}^{n+1}$ the connected component of $F\left(\widetilde{\Delta}^{n}\right) \cap \mathcal{V}$ containing $P^{n}$ (where $\left.\widetilde{\Delta}^{0}=\widetilde{\Delta}\right)$.

Definition 3.1. [The graph property]. Let $\Lambda$ be a $C^{1}$-submanifold of $N \times \mathbb{R}_{s}^{p} \times \mathbb{R}_{u}^{p}$. Let $B$ be an open ball in $\mathbb{R}_{u}^{p}$. We say that $\Lambda$ has the graph property over $B$, or equivalently that $\Lambda$ is $a$ graph over $B$, if there exists a $C^{1}$-map $\varpi: B \rightarrow N \times \mathbb{R}_{s}^{p}$ such that $\Lambda=\{(\varpi(u), u) ; u \in B\}$.

For $\delta$ small enough, we set $B_{\delta}:=\left\{u \in B_{u}^{p} ;\|u\|<\delta\right\}$ and $D_{\delta}:=\left\{(x, s, u) \in \mathcal{V} ; u \in B_{\delta}\right\}$. For $n \in \mathbb{N}$, we introduce the constant map

$$
\begin{aligned}
\ell_{n}: B_{\delta} & \longrightarrow N \times B_{s}^{p} \\
u & \longmapsto\left(P_{0}^{n}, 0\right)
\end{aligned}
$$

so that clearly $\widetilde{W}^{u u}\left(P_{0}^{n}\right) \cap D_{\delta}$ is the graph of $\ell_{n}$, for all $n \in \mathbb{N}$.

The $\lambda$-lemma in $V$ takes the following form.

Theorem 1. For all $n \in \mathbb{N}$, let $\widetilde{\Delta}^{n}$ and $\ell_{n}$ be as above. Then, there exists $\delta>0$ such that for all $n \in \mathbb{N}$, there exists a $C^{1}-m a p \xi_{n}: B_{\delta} \rightarrow N \times B_{s}^{p}$ such that $\widehat{\Delta}^{n}:=\widetilde{\Delta}^{n} \cap D_{\delta}$ is the graph of $\xi_{n}$. Moreover,

$$
\lim _{n \rightarrow \infty} d_{C^{1}}\left(\xi_{n}, \ell_{n}\right)=0
$$

where $d_{C^{1}}\left(\xi_{n}, \ell_{n}\right)=\sup _{u \in B_{\delta}}\left(d\left(\xi_{n}(u), \ell_{n}(u)\right)+\left\|\xi_{n}^{\prime}(u)-\ell_{n}^{\prime}(u)\right\|\right)$. 


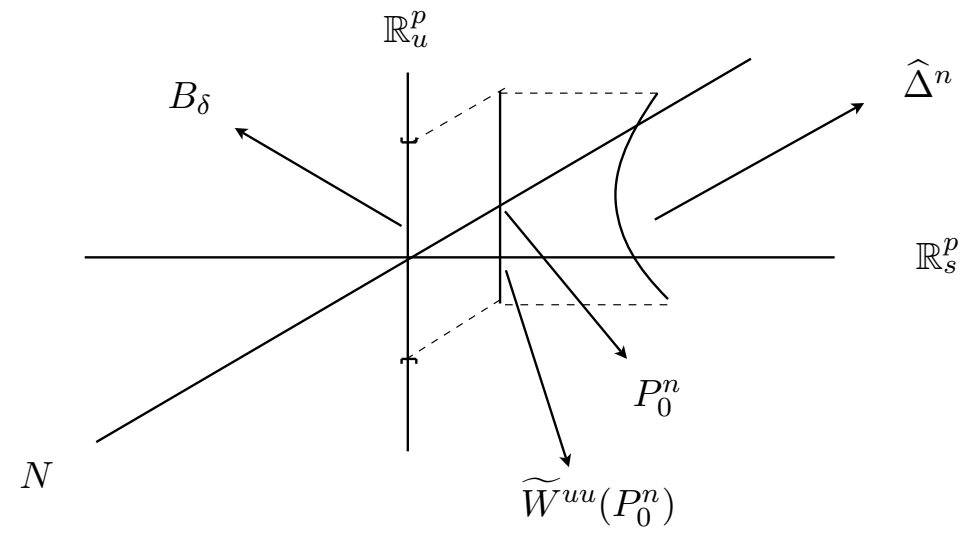

Figure 2: Graphs

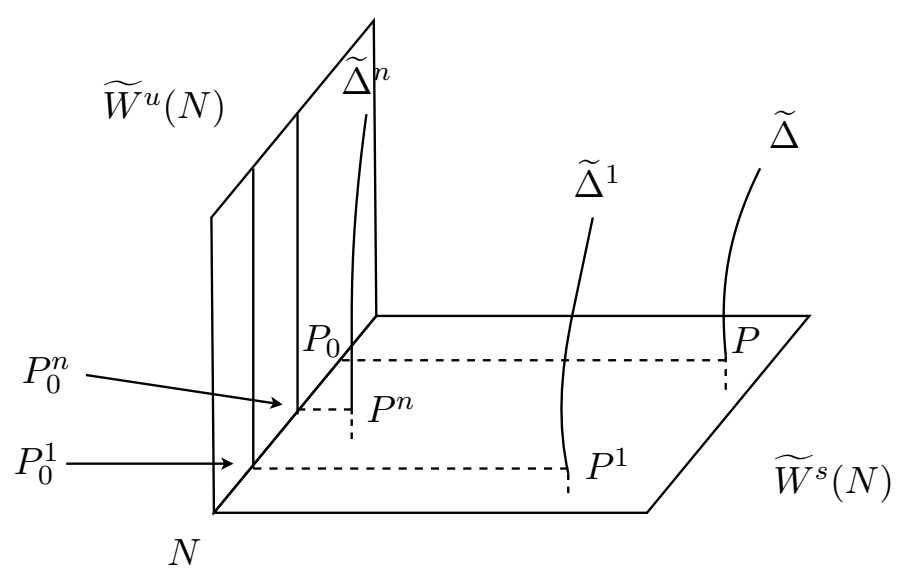

Figure 3: Straightening of $\widetilde{\Delta}$

We will need 4 steps to prove Theorem 1 in Section 4 . We will first show how, under iteration, arbitrary tangent vectors in $T_{P_{0}} \widetilde{\Delta}$ are straightened. We will then use the transversality of $\widetilde{\Delta}$ to $\widetilde{W}^{s}(N)$ to prove that some suitable part of $\widetilde{\Delta}$ (close to $P$ ) is a graph over a ball in $\mathbb{R}_{u}^{p}$. In the third step, we will show how this graph property is preserved under iteration over the same domain in $\mathbb{R}_{u}^{p}$. We will finally prove that tangent vectors along these graphs are straightened and a simple application of the Mean Value Theorem ends the proof of Theorem 1 .

We end this section with the definition of a notion of "closeness" for graphs which will be useful in the sequel.

Definition 3.2. We keep the notation of Theorem 1. Let $\varepsilon>0$ and $n \in \mathbb{N}$. We say that $\widehat{\Delta}^{n}$ and $\widetilde{W}^{u u}\left(P_{0}^{n}\right) \cap D_{\delta}$ are $C^{1} \varepsilon$-close if $d_{C^{1}}\left(\xi_{n}, \ell_{n}\right)<\varepsilon$.

\subsection{Theorem 2: in an arbitrary compact subset of $M$}

In this section, we introduce a new notion of graphs and convergence in the $C^{1}$ compact open topology (in a fixed relatively compact set in $M$ ).

Definition 3.3. Let $\mathcal{U}$ and $\bar{U}$ be two neighborhoods of $N$ in $M$ such that $\bar{U} \subset \mathcal{U}$. We suppose that there exists a $C^{2}$-diffeomorphism $\varphi: \mathcal{U} \longrightarrow N \times \mathbb{R}_{s}^{p} \times \mathbb{R}_{u}^{p}$. Let $m \in \mathbb{N}$ be fixed. We set 


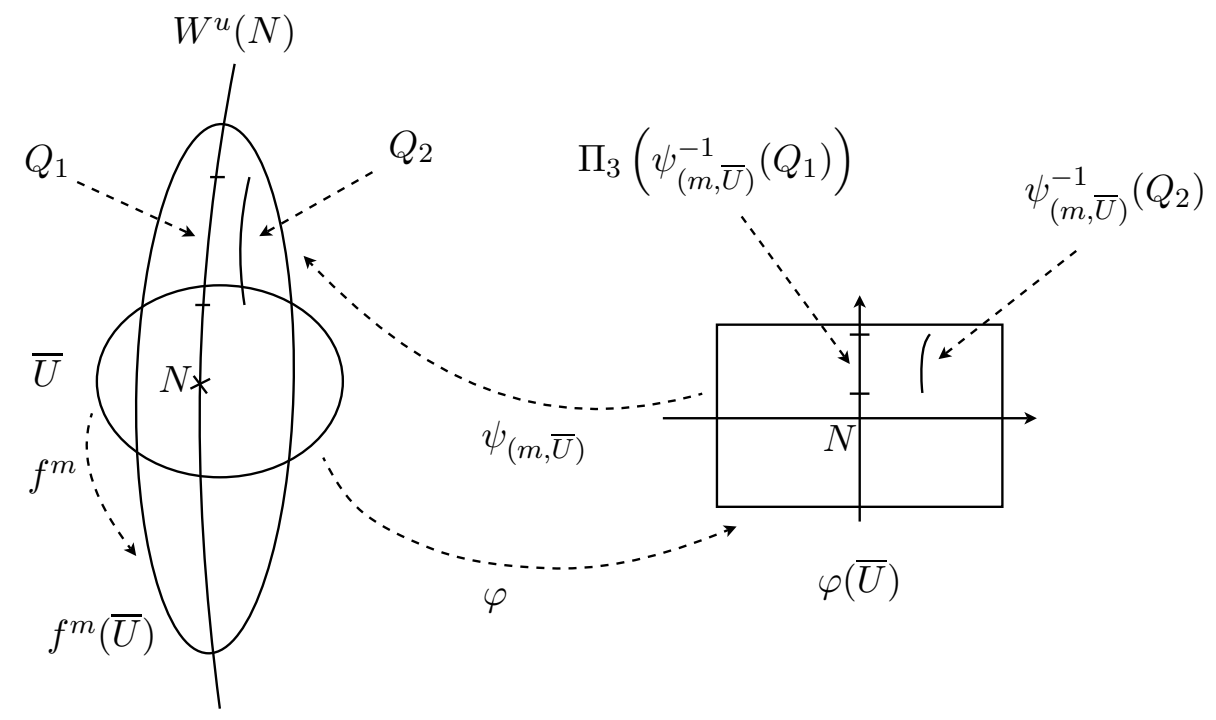

Figure 4: The $(m, \bar{U})$-graph property

$\psi_{(m, \bar{U})}:=\left.f^{m} \circ \varphi^{-1}\right|_{\varphi(\bar{U})}$. Let $Q_{1}$ be a $C^{1}$-submanifold of $M$ contained in $W^{u}(N) \cap f^{m}(\bar{U})$ and $Q_{2}$ be a $C^{1}$-submanifold of $M$ contained in $f^{m}(\bar{U})$. We say that $Q_{2}$ is a $(m, \bar{U})$-graph over $Q_{1}$ if $\psi_{(m, \bar{U})}^{-1}\left(Q_{2}\right)$ is a graph over $\Pi_{3}\left(\psi_{(m, \bar{U})}^{-1}\left(Q_{1}\right)\right)$ in the sense of Definition [3.1, where $\Pi_{3}$ denotes the projection over the third variable.

If $\psi_{(m, \bar{U})}^{-1}\left(Q_{2}\right)=\operatorname{graph} \xi=\operatorname{graph}(X, S)=\left\{(X(u), S(u), u) ; u \in \Pi_{3}\left(\psi_{1}^{-1}\left(Q_{1}\right)\right)\right\}$, we define the following distance

$$
d_{\left(C^{1}, m, \bar{U}\right)}\left(Q_{1}, Q_{2}\right):=\sup _{u \in \Pi_{3}\left(\psi_{(m, \bar{U})}^{-1}\left(Q_{1}\right)\right)} d\left(\psi_{(m, \bar{U})}(X(u), S(u), u), \psi_{(m, \bar{U})}(X(0), 0, u)\right)+
$$

$\sup _{\substack{u \in \Pi_{3}\left(\psi_{(m, \bar{U})}^{-1}\left(Q_{1}\right)\right) \\ v_{1} \in B_{\mathbb{R}_{u}^{p}}}}\left\|D \psi_{(m, \bar{U})}(X(u), S(u), u) \cdot\left(X^{\prime}(u) \cdot v_{1}, S^{\prime}(u) \cdot v_{1}, v_{1}\right)-D \psi_{(m, \bar{U})}(X(0), 0, u) \cdot\left(0, v_{1}\right)\right\|$ where $B_{\mathbb{R}_{u}^{p}}$ is the unit ball in $\mathbb{R}_{u}^{p}$.

We now state the global version of the $\lambda$-lemma.

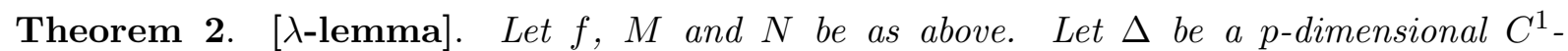
submanifold transversely intersecting $W^{s}(N)$ at some point $a$, and let $\Delta^{k}=f^{k}(\Delta)$, for $k \geq 1$. Let $a_{0}$ be the point in $N$ such that $a \in W^{s s}\left(a_{0}\right)$ and set $a_{0}^{k}:=f^{k}\left(a_{0}\right)$.

Then, there exist two neighborhoods $\mathcal{U}$ and $\bar{U}$ of $N$ in $M$ satisfying $\bar{U} \subset \mathcal{U}$, and a $C^{2}$ diffeomorphism $\varphi: \mathcal{U} \longrightarrow N \times \mathbb{R}_{s}^{p} \times \mathbb{R}_{u}^{p}$ such that $\forall m \in \mathbb{N}, \forall \varepsilon>0, \exists k_{0} \in \mathbb{N} ; \forall k \geq k_{0}$, there exists a $C^{1}$-submanifold $\bar{\Delta}^{k}$ in $f^{k}(\Delta) \cap f^{m}(\bar{U})$ such that $\bar{\Delta}^{k}$ is a $(m, \bar{U})$-graph over $W^{u u}\left(a_{0}^{k}\right) \cap f^{m}(\bar{U})$. Moreover,

$$
d_{\left(C^{1}, m, \bar{U}\right)}\left(\bar{\Delta}^{k}, W^{u u}\left(a_{0}^{k}\right) \cap f^{m}(\bar{U})\right)<\varepsilon .
$$

We devote Section [5 to the proof of Theorem 2. It will be a direct consequence of the proof of Theorem 1. 
Comments. Theorem 2 actually states the straightening property in any relatively compact set $K$ (with a non-empty interior) in $M$ intersecting all the unstable leaves of the submanifold $N$. More precisely, let $K$ be such a set. The sequence $\left(f^{m}(\bar{U}) \cap W^{u}(N)\right)_{m \in \mathbb{N}}$ is clearly an exhaustion of $W^{u}(N)$ by relatively compact sets. By definition of the unstable manifold, there exists an integer $m_{0}$ such that $W^{u}(N) \cap K \subset f^{m_{0}}(\bar{U})$. Then, one can easily prove that for all $\varepsilon>0$, there exists $k_{0} \in \mathbb{N}$ such that for all $k \geq k_{0}$, there exists a submanifold $\underline{\Delta}^{k}$ in $f^{k}(\Delta) \cap K$ such that $\underline{\Delta}^{k}$ is a $\left(m_{0}, \bar{U}\right)$-graph over $W^{u u}\left(a_{0}^{k}\right) \cap K$. Moreover,

$$
d_{\left(C^{1}, m_{0}, \bar{U}\right)}\left(\underline{\Delta}^{k}, W^{u u}\left(a_{0}^{k}\right) \cap K\right)<\varepsilon .
$$

Note that the convergence given by the basic $\lambda$-lemma is stronger than the Hausdorff one, for $\Delta$ and for its tangent space as well.

\section{Proof of Theorem 1}

In this section, we prove Theorem 1.

\subsection{General assumptions for Theorem 1}

Here we keep the notation of Proposition B and of Section 3.1 and we limit ourselves to the behavior of $F$ in $\mathcal{V}$. Recall that $\mathcal{V} \subset V=N \times B_{s}^{p} \times B_{u}^{p}$, where $B_{s, u}^{p}$ is an open ball centered at 0 in $\mathbb{R}^{p}$. Let $B_{s, u}^{p}$ be of radius $\varsigma$.

Since $\widetilde{W}^{s, u}(N)$ are invariant under $F$, then

$$
\begin{aligned}
& \forall x \in N, \forall s \in B_{s}^{p}, \quad F_{u}(x, s, 0)=0, \\
& \forall x \in N, \forall u \in B_{u}^{p}, \quad F_{s}(x, 0, u)=0 .
\end{aligned}
$$

In addition, $\forall x \in N, F(x, 0,0)=\left(F_{x}(x, 0,0), 0,0\right)$. Since the stable and unstable foliations are invariant, then for all $(x, s, u) \in \mathcal{V}$,

$$
F_{x}(x, 0, u)=F_{x}(x, s, 0)=F_{x}(x, 0,0) .
$$

Therefore, for $X=(x, 0,0) \in N \times\{(0,0)\}$, the derivative $D F(X)$ at $X$ can be represented as a diagonal matrix:

$$
D F(X)=\left(\begin{array}{ccc}
\partial_{x} F_{x}(X) & 0 & 0 \\
0 & \partial_{s} F_{s}(X) & 0 \\
0 & 0 & \partial_{u} F_{u}(X)
\end{array}\right) .
$$

The manifold $N \times\{(0,0)\}$ being normally hyperbolic for $F$, one can find a real number $\lambda \in] 0 ; 1[$ such that $\forall x \in N$,

$$
\begin{gathered}
\left\|\partial_{s} F_{s}(x, 0,0)\right\|<\lambda,\left\|\left(\partial_{u} F_{u}\right)^{-1}(x, 0,0)\right\|<\lambda,\left\|\partial_{s} F_{s}(x, 0,0)\right\| \cdot\left\|\left(\partial_{x} F_{x}(x, 0,0)\right)^{-1}\right\|<\lambda \\
\text { and }\left\|\partial_{x} F_{x}(x, 0,0)\right\| \cdot\left\|\left(\partial_{u} F_{u}(x, 0,0)\right)^{-1}\right\|<\lambda .
\end{gathered}
$$

Let $Y=(x, s, 0)$ be in $\widetilde{W}^{s}(N)$. Using Equations (4) and (6) , one easily sees that $D F(Y)$ takes the following form: 


$$
D F(Y)=\left(\begin{array}{ccc}
\partial_{x} F_{x}(Y) & 0 & \partial_{u} F_{x}(Y) \\
\partial_{x} F_{s}(Y) & \partial_{s} F_{s}(Y) & \partial_{u} F_{s}(Y) \\
0 & 0 & \partial_{u} F_{u}(Y)
\end{array}\right)
$$

One has an analogous property for the points of $\widetilde{W}^{u}(N)$.

We need to shrink $\mathcal{V}$ in order to have some estimates useful later on. Note first that $\mathcal{V}$ can be chosen so that $\partial_{x} F_{x}(Z)$ and $\partial_{u} F_{u}(Z)$ are invertible for all $Z \in \mathcal{V}$.

Let $\bar{\lambda}$ be in $] \lambda ; 1\left[\right.$. For simplicity, we choose $\bar{\lambda}=\frac{1+\lambda}{2}$. However, all the calculations in this proof can be adjusted so that they are compatible with any value of $\bar{\lambda} \in] \lambda ; 1\left[\right.$. Recall that $B_{s, u}^{p}$ is of radius $\varsigma$.

Proposition 4.1. For $\varsigma$ small enough, there exist real positive constants $C_{1}$ and $C_{2}$ such that for all $Z=(x, s, u) \in \mathcal{V}$, the following inequalities hold true

1. $\|s\|<\frac{5-5 \lambda}{2 C_{2}(11+\lambda)}$,

2. $\|D F(Z)\| \leq C_{1}$ and $\left\|D^{2} F(Z)\right\| \leq C_{2}$,

3. $\left\|\partial_{s} F_{s}(Z)\right\|<\bar{\lambda}$ and $\left\|\left[\partial_{u} F_{u}(Z)\right]^{-1}\right\|<\bar{\lambda}$,

4. $\left\|\partial_{x} F_{x}(Z)\right\| \cdot\left\|\left[\partial_{u} F_{u}(Z)\right]^{-1}\right\|<\bar{\lambda}$,

5. $\max \left(\left\|\partial_{s} F_{x}(Z)\right\|,\left\|\partial_{x} F_{s}(Z)\right\|\right)<\frac{5-5 \lambda}{2(11+\lambda)}$.

Proof. The proof is immediate because $F$ is at least $C^{2}$ and $\mathcal{V}$ is relatively compact. Note that the last item is immediate thanks to the form of $D F$ in Equation (7).

\subsection{Linear straightening of $T_{P^{m}} \widetilde{\Delta}^{m}$}

The following proposition states the straightening of the tangent space of $\widetilde{\Delta}$ at its base point, under iteration by $F$.

Proposition 4.2. For all $m \in \mathbb{N}$, the tangent space $T_{P^{m}} \widetilde{\Delta}^{m}$ is the graph of a linear map $L_{m}=\left(B_{m}, C_{m}\right): \mathbb{R}_{u}^{p} \longrightarrow T_{P_{0}^{m}} N \times \mathbb{R}_{s}^{p}$, whose norm satisfies:

$$
\lim _{m \rightarrow \infty}\left\|L_{m}\right\|=0 .
$$

Proof. We start with a quick study of the dynamics in $\widetilde{W}^{s}(N)$. Recall that $P=(x, s, 0)$ is the intersection point of $\widetilde{\Delta}$ and $\widetilde{W^{s}}(N)$. Note first that by Proposition 4.1, $\left\|\partial_{s} F_{s}\left(P^{i}\right)\right\|<\bar{\lambda}$, for all $i \geq 0$. For $i \geq 1$, we set $s_{i}:=F_{s}\left(P^{i-1}\right)$. Then by the Mean Value Theorem, one gets $\left\|s_{i}\right\| \leq \bar{\lambda}\left\|s_{i-1}\right\|$, and thus under iteration $\left\|s_{i}\right\| \leq \bar{\lambda}^{i}\|s\|$, that tends to 0 with an exponential speed.

- We will see now where the graph property appears. By transversality of $\widetilde{\Delta}$ and $\widetilde{W}^{s}(N)$, and since $\operatorname{dim} \widetilde{\Delta}=p, T_{P} \widetilde{\Delta}$ is the graph of a linear map defined on $\mathbb{R}_{u}^{p}$, with values in $T_{P_{0}} N \times \mathbb{R}_{s}^{p}$. More precisely, recall that $\Pi_{3}: N \times B_{s}^{p} \times B_{u}^{p} \longrightarrow B_{u}^{p}$ is the projection over the third variable. By transversality, $D \Pi_{\left.3\right|_{\tilde{\Delta}}}(P)$ is an isomorphism between $T_{P} \widetilde{\Delta}$ and $\mathbb{R}_{u}^{p}$. Therefore, there exist two linear maps $B$ and $C$ on $\mathbb{R}_{u}^{p}$, such that $T_{P} \widetilde{\Delta}$ is the image of the map

$$
(B, C, I): \mathbb{R}_{u}^{p} \longrightarrow T_{P_{0}} N \times \mathbb{R}_{s}^{p} \times \mathbb{R}_{u}^{p},
$$


where $I: \mathbb{R}_{u}^{p} \rightarrow \mathbb{R}_{u}^{p}$ is the identity map.

- Let us now see how the property of $T_{P} \widetilde{\Delta}$ being a graph of a linear map persists under iteration. We will proceed by induction. However, since the calculations are similar for all the iterates, we will content ourselves with detailing the proof for the first iteration.

The image of $T_{P} \widetilde{\Delta}$ under $D F(P)$ is $T_{F(P)} F(\widetilde{\Delta})$. For notational convenience, we will identify our linear maps with the matrices below (in the suitable algebras of linear applications) and the partial derivatives with the blocks in the matrices. For instance, $T_{F(P)} F(\Delta)$ is identified with the image of the linear map

$$
D F(P) \cdot\left(\begin{array}{c}
B \\
C \\
I
\end{array}\right): \mathbb{R}_{u}^{p} \longrightarrow T_{P_{0}^{1}} N \times \mathbb{R}_{s}^{p} \times \mathbb{R}_{u}^{p}
$$

Since $P$ lies in $\widetilde{W}^{s}(N)$, this is nothing but the image of the following map

$$
\left(\begin{array}{ccc}
\partial_{x} F_{x}(P) & 0 & \partial_{u} F_{x}(P) \\
\partial_{x} F_{s}(P) & \partial_{s} F_{s}(P) & \partial_{u} F_{s}(P) \\
0 & 0 & \partial_{u} F_{u}(P)
\end{array}\right)\left(\begin{array}{c}
B \\
C \\
I
\end{array}\right)=\left(\begin{array}{c}
\partial_{x} F_{x}(P) \cdot B+\partial_{u} F_{x}(P) \\
\partial_{x} F_{s}(P) \cdot B+\partial_{s} F_{s}(P) \cdot C+\partial_{u} F_{s}(P) \\
\partial_{u} F_{u}(P)
\end{array}\right)
$$

Since $\partial_{u} F_{u}(P): \mathbb{R}_{u}^{p} \longrightarrow \mathbb{R}_{u}^{p}$ is invertible, $T_{F(P)} F(\widetilde{\Delta})$, that is, $T_{P^{1}} \widetilde{\Delta}^{1}$ coincides with the image of

$$
\begin{gathered}
\left(\begin{array}{c}
\partial_{x} F_{x}(P) \cdot B+\partial_{u} F_{x}(P) \\
\partial_{x} F_{s}(P) \cdot B+\partial_{s} F_{s}(P) \cdot C+\partial_{u} F_{s}(P) \\
\partial_{u} F_{u}(P)
\end{array}\right) \cdot\left(\partial_{u} F_{u}(P)\right)^{-1}= \\
\left(\begin{array}{c}
\partial_{x} F_{x}(P) \cdot B \cdot\left(\partial_{u} F_{u}(P)\right)^{-1}+\partial_{u} F_{x}(P) \cdot\left(\partial_{u} F_{u}(P)\right)^{-1} \\
\partial_{x} F_{s}(P) \cdot B \cdot\left(\partial_{u} F_{u}(P)\right)^{-1}+\partial_{s} F_{s}(P) \cdot C \cdot\left(\partial_{u} F_{u}(P)\right)^{-1}+\partial_{u} F_{s}(P) \cdot\left(\partial_{u} F_{u}(P)\right)^{-1} \\
I
\end{array}\right) .
\end{gathered}
$$

This shows that $T_{P^{1}} \widetilde{\Delta}^{1}$ is also a graph. It is the image of the linear map

$$
\left(B_{1}, C_{1}, I\right): \mathbb{R}_{u}^{p} \longrightarrow T_{P_{0}^{1}} N \times \mathbb{R}_{s}^{p} \times \mathbb{R}_{u}^{p}
$$

where we have set

$$
B_{1}=\partial_{x} F_{x}(P) \cdot B \cdot\left(\partial_{u} F_{u}(P)\right)^{-1}+\partial_{u} F_{x}(P) \cdot\left(\partial_{u} F_{u}(P)\right)^{-1},
$$

and

$$
C_{1}=\partial_{x} F_{s}(P) \cdot B \cdot\left(\partial_{u} F_{u}(P)\right)^{-1}+\partial_{s} F_{s}(P) \cdot C \cdot\left(\partial_{u} F_{u}(P)\right)^{-1}+\partial_{u} F_{s}(P) \cdot\left(\partial_{u} F_{u}(P)\right)^{-1} .
$$

Pursuing the induction, one gets $B_{i}$ and $C_{i}(i>1)$, by applying $D F\left(P^{i-1}\right)$ to $T_{P^{i-1}} \widetilde{\Delta}^{i-1}$ (which is the image of $\left.\left(B_{i-1}, C_{i-1}, I\right)\right)$, and then normalizing by $\left(\partial_{u} F_{u}\left(P^{i-1}\right)\right)^{-1}$. We set $b_{i}=\left\|B_{i}\right\|$ and $c_{i}=\left\|C_{i}\right\|$, for $i \in \mathbb{N}$, where $B_{0}=B$ and $C_{0}=C$.

- To end the proof, it is enough now to prove that $\left(b_{i}\right)$ and $\left(c_{i}\right)$ converge to 0 . We begin with $\left(b_{i}\right)$. We fix an arbitrary $\varepsilon>0$. Proposition 4.1 yields, for all $i \in \mathbb{N}$,

$$
\left\|\partial_{x} F_{x}\left(P^{i}\right)\right\| \cdot\left\|\left(\partial_{u} F_{u}\left(P^{i}\right)\right)^{-1}\right\|<\bar{\lambda}
$$


so that, since $\left\|\left(\partial_{u} F_{u}\left(P^{i}\right)\right)^{-1}\right\|<1$,

$$
\left.b_{i+1} \leq\left\|\partial_{x} F_{x}\left(P^{i}\right)\right\| \cdot b_{i} \cdot\left\|\left(\partial_{u} F_{u}\left(P^{i}\right)\right)^{-1}\right\|+\| \partial_{u} F_{x}\left(P^{i}\right)\right)\|\cdot\|\left(\partial_{u} F_{u}\left(P^{i}\right)\right)^{-1} \| \leq \bar{\lambda} b_{i}+\beta_{i},
$$

where we have set $\left.\beta_{i}:=\| \partial_{u} F_{x}\left(P^{i}\right)\right) \|$. Therefore, for $n \in \mathbb{N}^{*}$,

$$
b_{n} \leq \bar{\lambda}^{n} b_{0}+\sum_{i=0}^{n-1} \bar{\lambda}^{i} \beta_{n-1-i} .
$$

Note that we are not interested in giving the optimal expression for the convergence. Since $\bar{\lambda}<1$, then for $n$ large enough, $\bar{\lambda}^{n} b_{0} \leq \frac{\varepsilon}{2}$. On the other hand, by the Mean Value Theorem, $\beta_{i}$ satisfies:

$$
\beta_{i} \leq C_{2} \bar{\lambda}^{i}\|s\|
$$

since $\left.\| \partial_{u} F_{x}\left(P_{0}^{i}\right)\right) \|=0$. As a consequence of Proposition 4.1, it is easy to see that $C_{2}\|s\| \leq 1$. Therefore

$$
\sum_{i=0}^{n-1} \bar{\lambda}^{i} \beta_{n-1-i} \leq \sum_{i=0}^{n-1} \bar{\lambda}^{i} \bar{\lambda}^{n-1-i} \leq \sum_{i=0}^{n-1} \bar{\lambda}^{n-1}=n \cdot \bar{\lambda}^{n-1} .
$$

Since $\bar{\lambda}<1$, then for $n$ large enough, $n . \bar{\lambda}^{n-1} \leq \frac{\varepsilon}{2}$. Then, for $n$ large enough, $b_{n} \leq \varepsilon$.

Note that one can also prove that the series $\sum b_{i}$ is convergent. This will be needed for the convergence of $\left(c_{i}\right)$.

Let us now study the convergence of the sequence $\left(c_{i}\right)$. For $i \geq 0$,

$$
\begin{aligned}
c_{i+1} & \leq\left\|\partial_{x} F_{s}\left(P^{i}\right)\right\| \cdot b_{i} \cdot\left\|\left(\partial_{u} F_{u}\left(P^{i}\right)\right)^{-1}\right\|+\left\|\partial_{s} F_{s}\left(P^{i}\right)\right\| \cdot c_{i} \cdot\left\|\left(\partial_{u} F_{u}\left(P^{i}\right)\right)^{-1}\right\| \\
& +\left\|\partial_{u} F_{s}\left(P^{i}\right)\right\| \cdot\left\|\left(\partial_{u} F_{u}\left(P^{i}\right)\right)^{-1}\right\| .
\end{aligned}
$$

It is easy to see, using the Mean Value Theorem, that $\left\|\partial_{x} F_{s}\left(P^{i}\right)\right\|<C_{2}\left\|s_{i}\right\|<1$, for all $i$. As we did for $\left(b_{i}\right)$, we define $\gamma_{i}:=\left\|\partial_{u} F_{s}\left(P^{i}\right)\right\|$ and get $\gamma_{i} \leq \bar{\lambda}^{i}$, following the same steps as for $\beta_{i}$. Therefore,

$$
c_{i+1} \leq b_{i}+\bar{\lambda} c_{i}+\bar{\lambda}^{i}
$$

and, for $n \geq 1$,

$$
c_{n} \leq \sum_{i=0}^{n-1} \bar{\lambda}^{(n-1-i)} b_{i}+\bar{\lambda}^{n} c_{0}+\sum_{i=0}^{n-1} \bar{\lambda}^{(n-1-i)} \bar{\lambda}^{i} .
$$

Since $\bar{\lambda}<1$, for $n$ large enough, one gets $\bar{\lambda}^{n} c_{0} \leq \frac{\varepsilon}{3}$. On the other hand, for $n$ large enough, $\sum_{i=0}^{n-1} \bar{\lambda}^{(n-1-i)} \cdot \bar{\lambda}^{i}=n \bar{\lambda}^{n-1} \leq \frac{\varepsilon}{3}$. Finally, let $s_{n-1}:=\sum_{i=0}^{n-1} \bar{\lambda}^{(n-1-i)} b_{i}$. Observe that $s_{n}$ is the general term of the Cauchy product of the series of general terms $b_{i}$ and $\bar{\lambda}^{i}$ respectively. These series are both convergent, so is their Cauchy product. Then $\left(s_{n}\right)$ converges to 0 . More precisely, for $n$ large enough, one has $s_{n-1} \leq \frac{\varepsilon}{3}$. This ends the proof of Proposition 4.2 ,

\subsection{The graph property for $\widetilde{\Delta}$}

We have seen above that, because of the transversality, $D \Pi_{3}(P)$ restricted to $T_{P} \widetilde{\Delta}$ is an isomorphism between $T_{P} \widetilde{\Delta}$ and $\mathbb{R}_{u}^{p}$. Then, by the Inverse Function Theorem, there exist a neighborhood $\mathcal{O}_{1}$ of $P$ in $\widetilde{\Delta}$ and a neighborhood $\mathcal{O}_{2}$ of 0 in $\mathbb{R}_{u}^{p}$ such that $\Pi_{\left.3\right|_{\tilde{\Delta}}}$ is a diffeomorphism from $\mathcal{O}_{1}$ onto $\mathcal{O}_{2}$. More precisely, there exists a real number $\widetilde{\delta}>0$ such that, if we set $B_{\widetilde{\delta}}:=\left\{u \in B_{u}^{p} ;\|u\|<\widetilde{\delta}\right\}$ and $D_{\widetilde{\delta}}:=\left\{(x, s, u) \in \mathcal{V} ; u \in B_{\widetilde{\delta}}\right\}$, then there exists a $C^{1}$-map $\xi: B_{\widetilde{\delta}} \rightarrow N \times B_{s}^{p}$, such that $\widetilde{\Delta} \cap D_{\widetilde{\delta}}$ is the graph of $\xi$ (in the sense of Definition 3.1). We set $\xi=(X, S)$. 


\subsection{The graph property for the iterates $\widetilde{\Delta}^{n}$ over a fixed strip}

We set $\widetilde{\nu}:=\left\|\xi^{\prime}\right\|=\max \left(\left\|X^{\prime}\right\|,\left\|S^{\prime}\right\|\right)=\sup _{u \in B_{\delta}}\left(\left\|\xi^{\prime}(u)\right\|\right)$ and $\nu:=\max (1, \widetilde{\nu})$. We will see later on why we choose $\nu$ (and not just $\widetilde{\nu}$ ) to bound the norm of all the derivatives of the graph maps. Let us set

$$
\varepsilon_{\nu}=\frac{1-\lambda}{12 \nu(1+\lambda)}=\frac{1-\bar{\lambda}}{12 \nu \bar{\lambda}}
$$

The reason behind this choice will be clarified later on. By uniform continuity, and due to the form of $D F$ on $\widetilde{W}^{s}(N)$ (Equation (8) ), there exists $\eta>0$, such that for all $(x, s, u) \in \mathcal{V}$, if $\|u\|<\eta$, then

$$
\left\|\partial_{x} F_{u}(x, s, u)\right\|<\varepsilon_{\nu} \text { and }\left\|\partial_{s} F_{u}(x, s, u)\right\|<\varepsilon_{\nu} .
$$

We then set

$$
\delta:=\min \left(1, \widetilde{\delta}, \eta, \frac{1-\bar{\lambda}}{3 C_{2}(2 \nu+1)^{2}}\right) .
$$

Proposition 4.3. Let $\delta$ and $\nu$ be as above. Then, for all $n \in \mathbb{N}$, there exists a $C^{1}$-map $\xi_{n}: B_{\delta} \rightarrow N \times B_{s}^{p}$ such that $\widehat{\Delta}^{n}:=\widetilde{\Delta}^{n} \cap D_{\delta}$ is the graph of $\xi_{n}$. Moreover, if for all $n \in \mathbb{N}$, $\xi_{n}=\left(X_{n}, S_{n}\right)$, then $\left\|\xi_{n}^{\prime}\right\|:=\max \left(\left\|X_{n}^{\prime}\right\|,\left\|S_{n}^{\prime}\right\|\right)=\sup _{u \in B_{\delta}}\left(\left\|\xi_{n}^{\prime}(u)\right\|\right)$ satisfies $\left\|\xi_{n}^{\prime}\right\| \leq \nu$.

The rest of this subsection is devoted to the proof of Proposition 4.3, We will proceed by induction. We first prove these statements for the first iteration, by using intermediate lemmas which will be very useful for the estimates later on. All the computations will be independent of $n$, which will easily yield the proof of the inductive step.

Note that when $n=0$, the statement follows from Section 4.3 and the definition of $\nu$. Therefore, we have to prove that if for $n \in \mathbb{N}, \widehat{\Delta}^{n}=\operatorname{graph} \xi_{n}=\left\{\left(X_{n}(u), S_{n}(u), u\right) ; u \in B_{\delta}\right\}$ with $\left\|\xi_{n}^{\prime}\right\| \leq \nu$, then $F\left(\widehat{\Delta}^{n}\right)$ is also the graph of a map $\xi_{n+1}$ over an open set in $\mathbb{R}_{u}^{p}$ strictly containing $B_{\delta}$. We then set

$$
\widehat{\Delta}^{n+1}=F\left(\widehat{\Delta}^{n}\right) \cap D_{\delta}=\widetilde{\Delta}^{n+1} \cap D_{\delta}=\operatorname{graph} \xi_{n+1}=\left\{\left(X_{n+1}(u), S_{n+1}(u), u\right) ; u \in B_{\delta}\right\} .
$$

Note that we will keep the same notation for $\xi_{n+1}$ and its restriction to $B_{\delta}$. We also have to prove that $\left\|\xi_{n+1}^{\prime}\right\|<\nu$.

To simplify this step and to keep our formulas legible, we will actually prove that Proposition 4.3 holds true when $n=1$. Since all the computations will be independent of $n$, one can easily see that the statements are valid for an arbitrary $n$.

By applying $F$ to $\widehat{\Delta}=\left\{(X(u), S(u), u) ; u \in B_{\delta}\right\}$, one gets

$$
F(\widehat{\Delta})=\left\{\left(F_{x}(X(u), S(u), u), F_{s}(X(u), S(u), u), F_{u}(X(u), S(u), u)\right) ; u \in B_{\delta}\right\} .
$$

Let $G(u):=F_{u}(X(u), S(u), u)=h$. We will prove that $G$ is a homeomorphism onto its image $B_{\delta}^{\prime}$ and that the latter strictly contains $B_{\delta}$. Then, it is easy to see that $F(\widehat{\Delta})$ restricted to $D_{\delta}^{\prime}:=\left\{(x, s, u) \in \mathcal{V} ; u \in B_{\delta}^{\prime}\right\}$ is the graph of $\left(X_{1}, S_{1}\right)$, where

$$
X_{1}(h)=F_{x}\left(X\left(G^{-1}(h)\right), S\left(G^{-1}(h)\right), G^{-1}(h)\right),
$$

and

$$
S_{1}(h)=F_{s}\left(X\left(G^{-1}(h)\right), S\left(G^{-1}(h)\right), G^{-1}(h)\right),
$$

for $h \in B_{\delta}^{\prime}$. We will need the following lemmas. 
Lemma 4.4. For all $u \in B_{\delta}, G^{\prime}(u)$ is an isomorphism on $\mathbb{R}_{u}^{p}$. Moreover,

$$
\left[G^{\prime}(u)\right]^{-1}=\left(\sum_{m \geq 0}(-H(u))^{m}\right) \cdot\left[\partial_{u} F_{u}(X(u), S(u), u)\right]^{-1},
$$

where $H(u):=\left[\partial_{u} F_{u}(X(u), S(u), u)\right]^{-1} \cdot\left[\partial_{x} F_{u}(X(u), S(u), u) \cdot X^{\prime}(u)+\partial_{s} F_{u}(X(u), S(u), u) \cdot S^{\prime}(u)\right]$. Proof. $G(u)=F_{u}(X(u), S(u), u)$ gives by derivation

$$
G^{\prime}(u)=\partial_{x} F_{u}(X(u), S(u), u) \cdot X^{\prime}(u)+\partial_{s} F_{u}(X(u), S(u), u) \cdot S^{\prime}(u)+\partial_{u} F_{u}(X(u), S(u), u) .
$$

Recall that the linear map $\partial_{u} F_{u}(x, s, u)$ is invertible for all $(x, s, u) \in \mathcal{V}$, and satisfies:

$$
\left\|\left[\partial_{u} F_{u}(x, s, u)\right]^{-1}\right\|<\bar{\lambda}<1 .
$$

Then one can write

$$
G^{\prime}(u)=\left[\partial_{u} F_{u}(X(u), S(u), u)\right] \cdot[I+H(u)],
$$

where $H(u):=\left[\partial_{u} F_{u}(X(u), S(u), u)\right]^{-1} \cdot\left[\partial_{x} F_{u}(X(u), S(u), u) \cdot X^{\prime}(u)+\partial_{s} F_{u}(X(u), S(u), u) \cdot S^{\prime}(u)\right]$. Since $\partial_{u} F_{u}(X(u), S(u), u)$ is invertible, it is enough to prove that $I+H(u)$ is invertible too. It is the case if $\|H(u)\|<1$ because it is an endomorphism of $\mathbb{R}_{u}^{p}$. So now we will prove that $\|H(u)\|<1$. It is easy to see that, by definition of $\varepsilon_{\nu}$ (equation (9)), for all $u \in B_{\delta}$,

$$
\|H(u)\|<2 \bar{\lambda} \nu \varepsilon_{\nu}=\frac{1-\bar{\lambda}}{6}<1 .
$$

Therefore $I+H(u)$ is invertible on $\mathbb{R}_{u}^{p}$ and $[I+H(u)]^{-1}=\sum_{m \geq 0}(-H(u))^{m}$. This ends the proof of Lemma 4.4 .

Lemma 4.5. For all $u \in B_{\delta}$, $\left\|\left[G^{\prime}(u)\right]^{-1}\right\|<1$.

Proof. This easily follows from the previous lemma. In fact,

$$
\begin{aligned}
\left\|\left[G^{\prime}(u)\right]^{-1}\right\| & \leq\left\|\sum_{m \geq 0}(-H(u))^{m}\right\| \cdot\left\|\left[\partial_{u} F_{u}(X(u), S(u), u)\right]^{-1}\right\| \\
& \leq \frac{1}{1-\|H(u)\|} \cdot\left\|\left[\partial_{u} F_{u}(X(u), S(u), u)\right]^{-1}\right\| \\
& <\frac{1}{1-2 \bar{\lambda} \nu \varepsilon_{\nu}} \cdot\left\|\left[\partial_{u} F_{u}(X(u), S(u), u)\right]^{-1}\right\| \\
& <\frac{6}{5+\bar{\lambda}} \cdot\left\|\left[\partial_{u} F_{u}(X(u), S(u), u)\right]^{-1}\right\|<\frac{6 \bar{\lambda}}{5+\bar{\lambda}}<1 .
\end{aligned}
$$

We will now prove that $G$ is invertible.

Proposition 4.6. There exists an open set $B_{\delta}^{\prime}$ in $\mathbb{R}_{u}^{p}$ strictly containing $B_{\delta}$, such that $G$ is a homeomorphism from $B_{\delta}$ onto $B_{\delta}^{\prime}$.

Proof. Without loss of generality, we can assume that $\xi$ is defined on $\bar{B}_{\delta}$. We introduce an auxiliary map defined on $\bar{B}_{\delta}$,

$$
\chi(u)=\left[\partial_{u} F_{u}(X(0), S(0), 0)\right]^{-1} \cdot G(u)=\left[\partial_{u} F_{u}(P)\right]^{-1} \cdot G(u) .
$$

We will first study the invertibility of $\chi$, from which that of $G$ easily follows. Let $y$ be in a subset of $\mathbb{R}_{u}^{p}$ to be specified later on. We are looking for the conditions under which there exists a unique $x \in B_{\delta}$, such that $y=\chi(x)$. We let $\psi(x):=x-\chi(x)+y$, so that the point $y$ has a unique preimage under $\chi$ if and only if $\psi$ has a unique fixed point. To prove this last property, we will need the next lemma. 
Lemma 4.7. For all $u \in \bar{B}_{\delta},\left\|I-\chi^{\prime}(u)\right\|<\frac{1-\bar{\lambda}}{2}$.

Proof. By derivating $\chi(u)=\left[\partial_{u} F_{u}(P)\right]^{-1} \cdot G(u)$, one gets

$$
\chi^{\prime}(u)=\left[\partial_{u} F_{u}(P)\right]^{-1} \cdot\left[\partial_{u} F_{u}(X(u), S(u), u)\right] \cdot[I+H(u)] .
$$

We set $\mathcal{W}:=\left[\partial_{u} F_{u}(P)\right]^{-1} \cdot\left[\partial_{u} F_{u}(X(u), S(u), u)\right]$ and $\mathcal{T}:=\mathcal{W}-I$ so that $\mathcal{W}=\mathcal{T}+I$ and $\chi^{\prime}(u)-I=\mathcal{W} \cdot(I+H(u))-I$. Recall that $\|H(u)\|<2 \bar{\lambda} \nu \varepsilon_{\nu}=\frac{1-\bar{\lambda}}{6}$ (see the proof of Lemma 4.4). Therefore,

$$
\begin{aligned}
\|\mathcal{T}\| & =\left\|\left[\partial_{u} F_{u}(P)\right]^{-1} \cdot\left[\partial_{u} F_{u}(X(u), S(u), u)\right]-I\right\| \\
& =\left\|\left[\partial_{u} F_{u}(P)\right]^{-1} \cdot\left[\partial_{u} F_{u}(X(u), S(u), u)-\partial_{u} F_{u}(P)\right]\right\| \\
& \leq\left\|\left[\partial_{u} F_{u}(P)\right]^{-1}\right\| \cdot \|\left[\partial_{u} F_{u}(X(u), S(u), u)-\partial_{u} F_{u}(X(0), S(0), 0) \|\right. \\
& \leq \bar{\lambda}\left(2 C_{2} \nu+C_{2}\right)\|u\|,
\end{aligned}
$$

by the Mean Value Theorem. Writing $\chi^{\prime}(u)-I=(\mathcal{T}+I) .(I+H(u))-I=H(u)+\mathcal{T} .(I+H(u))$ gives

$$
\begin{aligned}
\left\|\chi^{\prime}(u)-I\right\| & \leq\|H(u)\|+\|\mathcal{T}\| \cdot(1+\|H(u)\|) \\
& <2 \bar{\lambda} \nu \varepsilon_{\nu}+\bar{\lambda}\left(2 C_{2} \nu+C_{2}\right)\|u\|\left(1+2 \varepsilon_{\nu} \bar{\lambda} \nu\right) \\
& <\frac{1-\bar{\lambda}}{6}+C_{2}(2 \nu+1)^{2}\|u\|,
\end{aligned}
$$

because $\bar{\lambda}<1$ and $\bar{\lambda} \varepsilon_{\nu}<1$ using equation (9).

Recall that $\|u\|<\frac{1-\bar{\lambda}}{3 C_{2}(2 \nu+1)^{2}}$ by equation (11), which yields

$$
\begin{aligned}
\left\|\chi^{\prime}(u)-I\right\| & <\frac{1-\bar{\lambda}}{6}+\frac{1-\bar{\lambda}}{3} \\
& <\frac{1-\bar{\lambda}}{2} .
\end{aligned}
$$

This ends the proof of Lemma 4.7.

- We now go back to proving the invertibility of $\chi$. Let $\kappa:=\frac{1-\bar{\lambda}}{2}$. Clearly $\kappa<1$. The last lemma shows that $\psi=I_{B_{\delta}}-\chi+y$ is a contracting map. In order for it to have a unique fixed point, one needs to have $\psi\left(\bar{B}_{\delta}\right) \subset \bar{B}_{\delta}$. And this condition is satisfied if $\|y\| \leq \delta(1-\kappa)$. Therefore $\chi: \bar{B}_{\delta} \longrightarrow \operatorname{Im} \chi$ is bijective and satisfies $\bar{B}_{\delta(1-\kappa)} \subset \operatorname{Im} \chi$.

- The invertibility of $G$ easily follows from that of $\chi$. Recall that $\chi=\left[\partial_{u} F_{u}(P)\right]^{-1}$.G. Therefore, $G: \bar{B}_{\delta} \longrightarrow \operatorname{Im} G$ is an homeomorphism and satisfies $B_{\delta}^{\prime}:=\operatorname{Im} G \supset \bar{B}_{\frac{\delta(1-\kappa)}{\bar{\lambda}}}$.

Recall that $\kappa=\frac{1-\bar{\lambda}}{2}$ which gives $(1-\kappa)>\bar{\lambda}$ and thus $B_{\delta}^{\prime}$, which contains $B_{\frac{\delta(1-\kappa)}{\bar{\lambda}}}$, strictly contains $B_{\delta}$. This ends the proof of the proposition.

Therefore, the proof of the graph property in Proposition 4.3 for the case $n=1$ is complete. Let $\widehat{\Delta}^{1}=F(\widehat{\Delta}) \cap D_{\delta}=\operatorname{graph} \xi_{1}=\left\{\left(X_{1}(u), S_{1}(u), u\right) ; u \in B_{\delta}\right\}$. The next proposition will not only end the proof of the case $n=1$, but will also be a preliminary step to estimating $\lim _{n \rightarrow \infty}\left\|\xi_{n}^{\prime}\right\|$ in Section 4.5,

Proposition 4.8. If we set $\left\|\xi_{1}^{\prime}\right\|:=\sup _{u \in B_{\delta}}\left(\left\|\xi_{1}^{\prime}(u)\right\|\right)=\max \left(\left\|X_{1}^{\prime}\right\|,\left\|S_{1}^{\prime}\right\|\right)$, then $\left\|\xi_{1}^{\prime}\right\|<\nu$.

Proof. We recall that for $h \in B_{\delta}^{\prime}$,

$$
X_{1}(h)=F_{x}\left(X\left(G^{-1}(h)\right), S\left(G^{-1}(h)\right), G^{-1}(h)\right),
$$


and

$$
S_{1}(h)=F_{s}\left(X\left(G^{-1}(h)\right), S\left(G^{-1}(h)\right), G^{-1}(h)\right) .
$$

Since we are only interested in uniform norms over $B_{\delta}$, we consider $h$ to belong to $B_{\delta}$ from now on. We let $u:=G^{-1}(h)$. Then $u \in G^{-1}\left(B_{\delta}\right) \varsubsetneqq B_{\delta}$. We write

$$
X_{1}(G(u))=F_{x}(X(u), S(u), u),
$$

and

$$
S_{1}(G(u))=F_{s}(X(u), S(u), u) .
$$

By derivating the two sides with respect to $u$ and inverting $G^{\prime}(u)$, one gets for all $u \in B_{\delta}$

$$
\begin{aligned}
X_{1}^{\prime}(G(u)) & =\partial_{x} F_{x}(X(u), S(u), u) \cdot X^{\prime}(u) \cdot\left[G^{\prime}(u)\right]^{-1}+\partial_{s} F_{x}(X(u), S(u), u) \cdot S^{\prime}(u) \cdot\left[G^{\prime}(u)\right]^{-1} \\
& +\partial_{u} F_{x}(X(u), S(u), u) \cdot\left[G^{\prime}(u)\right]^{-1},
\end{aligned}
$$

and

$$
\begin{aligned}
S_{1}^{\prime}(G(u)) & =\partial_{x} F_{s}(X(u), S(u), u) \cdot X^{\prime}(u) \cdot\left[G^{\prime}(u)\right]^{-1}+\partial_{s} F_{s}(X(u), S(u), u) \cdot S^{\prime}(u) \cdot\left[G^{\prime}(u)\right]^{-1} \\
& +\partial_{u} F_{s}(X(u), S(u), u) \cdot\left[G^{\prime}(u)\right]^{-1} \cdot
\end{aligned}
$$

Let us begin by studying $T:=\left\|\partial_{x} F_{x}(X(u), S(u), u)\right\| .\left\|\left[G^{\prime}(u)\right]^{-1}\right\|$. Using the estimates in Lemma 4.5, one gets

$$
\begin{aligned}
T & \leq\left\|\partial_{x} F_{x}(X(u), S(u), u)\right\| \cdot\left\|[I+H(u)]^{-1}\right\| \cdot\left\|\left[\partial_{u} F_{u}(X(u), S(u), u)\right]^{-1}\right\| \\
& <\bar{\lambda} \cdot\left\|[I+H(u)]^{-1}\right\|<\frac{6 \bar{\lambda}}{5+\bar{\lambda}}:=\widetilde{\alpha},
\end{aligned}
$$

where we can easily see that $0<\bar{\lambda}<\widetilde{\alpha}<1$. Recall that by Proposition 4.1, for all $u \in B_{\delta}$,

$$
\max \left(\left\|\partial_{s} F_{x}(X(u), S(u), u)\right\|,\left\|\partial_{x} F_{s}(X(u), S(u), u)\right\|\right)<\frac{5-5 \lambda}{2(11+\lambda)},
$$

which yields

$$
\left\|\xi_{1}^{\prime}\right\|<\left(\widetilde{\alpha}+\frac{5-5 \lambda}{2(11+\lambda)}\right)\left\|\xi^{\prime}\right\|+\sup _{u \in B_{\delta}} \max \left(\left\|\partial_{u} F_{x}(X(u), S(u), u)\right\|,\left\|\partial_{u} F_{s}(X(u), S(u), u)\right\|\right) .
$$

On the one hand, $\widetilde{\alpha}=\frac{6 \bar{\lambda}}{5+\bar{\lambda}}=\frac{6+6 \lambda}{11+\lambda}$, and thus $\widetilde{\alpha}+\frac{5-5 \lambda}{2(11+\lambda)}=\frac{1+\widetilde{\alpha}}{2}:=\beta$, with $0<\widetilde{\alpha}<\beta<1$. On the other hand, using the particular form of $F$ on the unstable manifold (Equations (5) and (6) ), for $X \in \widetilde{W}^{u}(N)$, the derivative $D F(X)$ at $X$ has the following form

$$
D F(X)=\left(\begin{array}{ccc}
\partial_{x} F_{x}(X) & \partial_{s} F_{x}(X) & 0 \\
0 & \partial_{s} F_{s}(X) & 0 \\
\partial_{x} F_{u}(X) & \partial_{s} F_{u}(X) & \partial_{u} F_{u}(X)
\end{array}\right) .
$$

Therefore, using this particular form and the Mean Value Theorem, one gets

$$
\sup _{u \in B_{\delta}} \max \left(\left\|\partial_{u} F_{x}(X(u), S(u), u)\right\|,\left\|\partial_{u} F_{s}(X(u), S(u), u)\right\|\right) \leq C_{2} \sup _{u \in B_{\delta}}\|S(u)\|,
$$

and so

$$
\left\|\xi_{1}^{\prime}\right\|<\beta\left\|\xi^{\prime}\right\|+C_{2} \sup _{u \in B_{\delta}}\|S(u)\| .
$$

Using item 1 of Proposition 4.1, and the fact that $\nu \geq 1$, one gets

$$
\left\|\xi_{1}^{\prime}\right\|<\left(\beta+\frac{5-5 \lambda}{2(11+\lambda)}\right) \nu=\nu
$$

This ends the proof of the lemma. 
Observe that the fact that $\nu$ is larger than 1 is crucial to show that $\left\|\xi_{1}^{\prime}\right\|<\nu$ which explains our initial choice in the beginning of Section 4.4. Since all the computations in the previous lemmas are independent of $n$, the proof of the inductive step easily follows.

We then set $\widehat{\Delta}^{n}=\widetilde{\Delta}^{n} \cap D_{\delta}=\operatorname{graph} \xi_{n}=\left\{\left(X_{n}(u), S_{n}(u), u\right) ; u \in B_{\delta}\right\}$ for all $n \in \mathbb{N}$. This ends the proof of Proposition 4.3 .

\subsection{Linear straightening along the graphs}

We will now see how tangent vectors along the graphs are straightened. We will use the estimates of the previous section to prove the following proposition.

Recall that $\widehat{\Delta}^{n}=\operatorname{graph} \xi_{n}=\operatorname{graph}\left(X_{n}, S_{n}\right)=\left\{\left(X_{n}(u), S_{n}(u), u\right) ; u \in B_{\delta}\right\}$ for all $n \in \mathbb{N}$.

Proposition 4.9. For all $\varepsilon>0$, there exists $n_{0} \in \mathbb{N}$, such that for all $n \geq n_{0},\left\|\xi_{n}^{\prime}\right\|<\varepsilon$.

Proof. Generalizing to all the iterates Inequality (13), since the estimates are uniform with respect to the order of the iteration, one gets

$$
\left\|\xi_{n+1}^{\prime}\right\|<\beta\left\|\xi_{n}^{\prime}\right\|+C_{2} \sup _{u \in B_{\delta}}\left\|S_{n}(u)\right\| .
$$

By the Mean Value Theorem, one can prove by induction that $\sup _{u \in B_{\delta}}\left\|S_{n}(u)\right\| \leq \bar{\lambda}^{n} \sup _{u \in B_{\delta}}\|S(u)\|$. More precisely, for all $u \in B_{\delta}$ and for all $n \in \mathbb{N}^{*}$, there exists $Z=\left(Z_{1}, Z_{2}, Z_{3}\right) \in \widehat{\Delta}^{n-1}$ such that $S_{n}(u)=F_{s}(Z)=F_{s}(Z)-F_{s}\left(Z_{1}, 0, Z_{3}\right)$. Therefore $\left\|S_{n}(u)\right\| \leq \bar{\lambda}\left\|Z_{2}\right\| \leq \bar{\lambda} \sup _{u \in B_{\delta}}\left\|S_{n-1}(u)\right\|$, since $\widehat{\Delta}^{n-1}=\operatorname{graph}\left(X_{n-1}, S_{n-1}\right)$, which proves our claim. Since $C_{2} \sup _{u \in B_{\delta}}\|S(u)\|<1$, then by Inequality (14),

$$
\left\|\xi_{n+1}^{\prime}\right\|<\beta\left\|\xi_{n}^{\prime}\right\|+\bar{\lambda}^{n} .
$$

The proof of the convergence follows the same lines as that of $\left(b_{n}\right)$ in Section 4.2 , since $\beta<1$.

\subsection{Nonlinear straightening and proof of Theorem 1}

We can now end the proof of Theorem 1 by a simple application of the Mean Value Theorem. We get for $n \geq n_{0}$,

$$
\begin{aligned}
\sup _{u \in B_{\delta}} d\left(\xi_{n}(u),\left(P_{0}^{n}, 0\right)\right) & \leq \sup _{u \in B_{\delta}} d\left(\xi_{n}(u), \xi_{n}(0)\right)+d\left(\xi_{n}(0),\left(P_{0}^{n}, 0\right)\right) \\
& <\varepsilon+\left\|S_{n}(0)\right\| \\
& <\varepsilon+\bar{\lambda}^{n}\|S(0)\|,
\end{aligned}
$$

where we have used that $\|u\|<1$. The convergence easily follows. This completes the proof of Theorem 1.

\section{Proof of Theorem 2}

We will now prove Theorem 2 which will be a consequence of Theorem 1 . Let $\varphi$ be the diffeomorphism given by Proposition $\mathrm{B}$ and $\mathcal{U}$ be as in Section [3.1. Let $\delta$ be given by Theorem 1. We set $\bar{U}:=\varphi^{-1}\left(D_{\delta}\right)$. Let $m \in \mathbb{N}$ be fixed, then $\psi_{(m, \bar{U})}=\left.f^{m} \circ \varphi^{-1}\right|_{D_{\delta}}$. Let $\left(\widehat{\Delta}^{n}\right)$ be as in Theorem 1. For all $k \geq m$, let $\bar{\Delta}^{k}:=\psi_{(m, \bar{U})}\left(\widehat{\Delta}^{k-m}\right)$. The $(m, \bar{U})$-graph property of $\bar{\Delta}^{k}$ is an immediate consequence of Theorem 1. As for the convergence, the $C^{0}$ part 


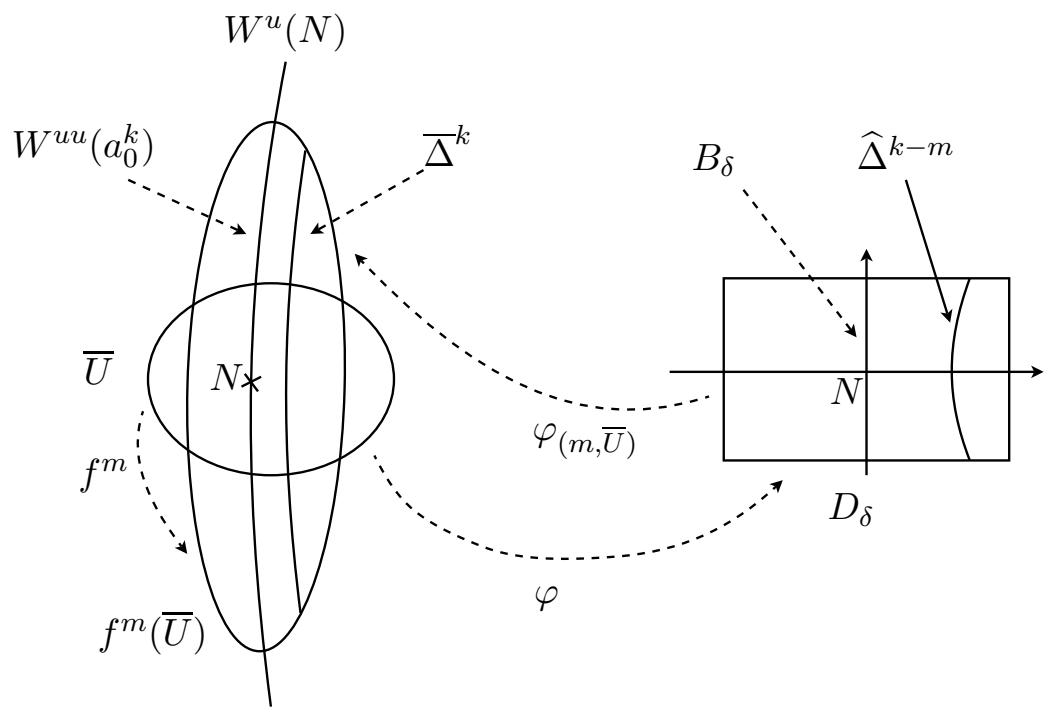

Figure 5: In $f^{m}(\bar{U})$

of the convergence is obvious by uniform continuity of $\psi_{(m, \bar{U})}$. It is now enough to prove the convergence of the second term of the $\left(C^{1}, m, \bar{U}\right)$-distance. There exist two positive real numbers $\bar{C}$ and $\overline{\bar{C}}$, such that for all $u \in B_{\delta}$, for all $v_{1} \in B_{\mathbb{R}_{u}^{p}}$, for all $n \in \mathbb{N}$, if we set $T:=\left\|D \psi_{(m, \bar{U})}\left(\xi_{n}(u), u\right) \cdot\left(\xi_{n}{ }^{\prime}(u) \cdot v_{1}, v_{1}\right)-D \psi_{(m, \bar{U})}\left(X_{n}(0), 0, u\right) \cdot\left(0, v_{1}\right)\right\|$, then

$$
\begin{aligned}
T \leq & \left\|D \psi_{(m, \bar{U})}\left(\xi_{n}(u), u\right) \cdot\left(\xi_{n}{ }^{\prime}(u) \cdot v_{1}, v_{1}\right)-D \psi_{(m, \bar{U})}\left(\xi_{n}(u), u\right) \cdot\left(0, v_{1}\right)\right\| \\
& +\left\|D \psi_{(m, \bar{U})}\left(\xi_{n}(u), u\right) \cdot\left(0, v_{1}\right)-D \psi_{(m, \bar{U})}\left(X_{n}(0), 0, u\right) \cdot\left(0, v_{1}\right)\right\| \\
\leq & \left\|D \psi_{(m, \bar{U})}\left(\xi_{n}(u), u\right)\right\| \cdot\left\|\xi_{n}{ }^{\prime}(u)\right\|+\left\|D \psi_{(m, \bar{U})}\left(\xi_{n}(u), u\right)-D \psi_{(m, \bar{U})}\left(X_{n}(0), 0, u\right)\right\| \\
\leq & \bar{C} \cdot\left\|\xi_{n}{ }^{\prime}(u)\right\|+\bar{C} d\left(\left(\xi_{n}(u), u\right),\left(X_{n}(0), 0, u\right)\right)
\end{aligned}
$$

by the Mean Value Theorem. The convergence follows from Theorem 1. By setting $n:=k-m$, the proof of Theorem 2 is now complete.

\section{Application to diffusion}

We will now use the $\lambda$-lemma to prove a diffusion result. We will prove the existence of a shadowing orbit for a finite family of invariant dynamically minimal sets contained in a normally hyperbolic manifold and having successive heteroclinic connections. We will see that the existence of Arnold's diffusion orbit easily follows from this application.

\subsection{Shadowing orbits for a finite family of invariant minimal sets}

In this section, we prove a corollary of the $\lambda$-lemma that gives the existence of a shadowing orbit for a transition chain. Let $f, M$ and $N$ be as in Section 3 . If $A$ is an invariant dynamically minimal set contained in $N$, that is, a set in which the orbit of each point is dense, we set

$$
W^{u}(A):=\bigcup_{a \in A} W^{u u}(a)
$$

Definition 6.1. [Transition chain]. Let $n \in \mathbb{N},(n>1)$. Let $\left(A_{k}\right)_{1 \leq k \leq n}$ be a finite family of invariant dynamically minimal sets contained in $N$. We say that $\left(A_{k}\right)$ is a transition chain if, for all $k=1, \ldots, n-1, W^{u}\left(A_{k}\right) \cap W^{s}\left(A_{k+1}\right) \neq \emptyset$. 


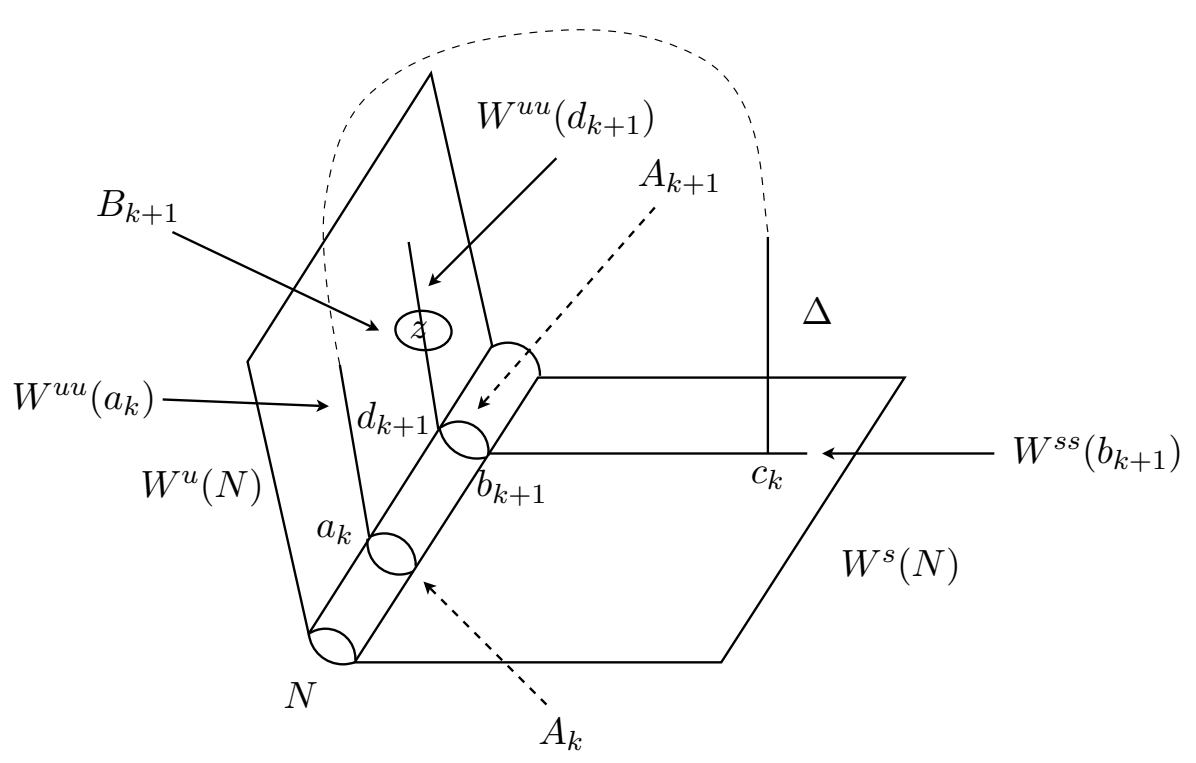

Figure 6: Heteroclinic connections

Note that we do not require any regularity for the sets. In the Hamiltonian nearly integrable case, they can be general Aubry-Mather sets for instance.

We will only need the convergence in the $C^{0}$ topology stated in the $\lambda$-lemma to prove the following result. In Figure [6, we illustrate the assumptions of Corollary 6.2, in the particular case $n_{0}=2$ and $p=1$. Of course, since the invariant manifolds are 3-dimensional, this is only a rough representation of the situation.

Corollary 6.2. Let $f, M$ and $N$ be as in Section 3. Let $\left(A_{k}\right)_{1 \leq k \leq n}$ be a transition chain in $N$ such that, for all $k=1, \ldots, n-1$, there exist $a_{k} \in A_{k}, b_{k+1} \in A_{k+1}$ and $c_{k} \in W^{u u}\left(a_{k}\right) \cap W^{s s}\left(b_{k+1}\right)$ such that $W^{u u}\left(a_{k}\right)$ and $W^{s}(N)$ transversely intersect at $c_{k}$. Then, for any $\varrho>0$, there exists an orbit $\Gamma$ such that, for all $k=1, \ldots, n, \Gamma$ intersects the $\varrho$-neighborhood of $A_{k}$.

Proof. We fix $\varrho>0$ and we denote by $\mathcal{V}_{\varrho}\left(A_{k}\right)$ the $\varrho$-neighborhood of $A_{k}$ for all $k=1, \ldots, n$. Without any loss of generality, we can suppose that, for all $k=1, \ldots, n, \mathcal{V}_{\varrho}\left(A_{k}\right) \subset \mathcal{U}$ (defined in Section 3.1). Therefore, we can restrict the problem to the straightening neighborhood $\mathcal{V} \subset V$ (see Proposition B and Proposition 4.1).

Fix $k=1, \ldots, n-1$ and fix a ball $B_{k+1}$ centered at some point $z$ of $W^{u}\left(A_{k+1}\right)$ and contained in $\mathcal{V}_{\varrho}\left(A_{k+1}\right)$. Since $z$ is in $W^{u}\left(A_{k+1}\right)$, there exists a unique $d_{k+1} \in A_{k+1}$ such that $z \in W^{u u}\left(d_{k+1}\right)$. Let us set $\Delta:=W^{u u}\left(a_{k}\right)$, then $\Delta$ is an immersed $p$-dimensional $C^{3}$-submanifold of $M$, transversely intersecting $W^{s}(N)$. For $m \in \mathbb{N}^{*}$, we let $b_{k+1}^{m}:=F^{m}\left(b_{k+1}\right)$ and $\Delta^{m}$ be the connected component of $F\left(\Delta^{m-1}\right) \cap \mathcal{V}$ containing $c_{k}^{m}:=F^{m}\left(c_{k}\right)$.

- By the $\lambda$-lemma, for all $\varepsilon>0$, there exists $N_{1} \in \mathbb{N}$ such that for all $m \geq N_{1}, \Delta^{m}$ is $\varepsilon$-close to $W^{u u}\left(b_{k+1}^{m}\right)$ (in the sense of Definition 3.2).

- Since $A_{k+1}$ is invariant, the sequence $\left(b_{k+1}^{m}\right)_{m \in \mathbb{N}}$ lies in $A_{k+1}$. Since this set is also minimal, we can extract a subsequence $\left(b_{k+1}^{m_{j}}\right)_{j \in \mathbb{N}}$ such that $\lim _{j \rightarrow \infty} b_{k+1}^{m_{j}}=d_{k+1}$. More precisely,

$$
\forall \varepsilon>0, \exists N_{2} \in \mathbb{N} ; j \geq N_{2} \Rightarrow d\left(b_{k+1}^{m_{j}}, d_{k+1}\right)<\varepsilon .
$$

- The foliations being straightened, for $j$ large enough, $W^{u u}\left(b_{k+1}^{m_{j}}\right)$ is $\varepsilon$-close to $W^{u u}\left(d_{k+1}\right)$. 
- Therefore for $j$ large enough, $\Delta^{m_{j}}$ intersects $B_{k+1}$.

Let $y$ be in $\Delta^{m_{j}} \cap B_{k+1}$ which is in $W^{u}\left(A_{k}\right)$. Then, for $q$ large enough, $F^{-q}(y) \in \mathcal{V}_{\varrho}\left(A_{k}\right)$. Therefore there exists a ball $B_{k}$ centered at $F^{-q}(y)$ and contained in $\mathcal{V}_{\varrho}\left(A_{k}\right)$ such that

$$
F^{q}\left(B_{k}\right) \subset B_{k+1}
$$

We proved then the existence of a ball $B_{k}$ centered on $W^{u}\left(A_{k}\right)$ in $\mathcal{V}_{\varrho}\left(A_{k}\right)$ and a positive $q$ such that $F^{q}\left(B_{k}\right) \subset B_{k+1}$.

Therefore, given a ball $B_{n}$ centered on $W^{u}\left(A_{n}\right)$ and contained in $\mathcal{V}_{\varrho}\left(A_{n}\right)$, an immediate induction proves the existence of an integer $q^{*}$ and a ball $B_{1}$ centered on $W^{u}\left(A_{1}\right)$ and contained in $\mathcal{V}_{\varrho}\left(A_{1}\right)$, whose sequence of iterates intersects each $\mathcal{V}_{\varrho}\left(A_{k}\right)$ and which moreover satisfies $F^{q^{*}}\left(B_{1}\right) \subset B_{n}$. This proves our claim.

\subsection{Particular case: Arnold's example}

We will see in this section that Arnold's system (Arn64) satisfies all the assumptions of Corollary 6.2 and thus, one easily deduces the existence of drifting orbits. In Arnold's example, the stable manifold of a torus transversely intersects the unstable manifold of the next torus. These manifolds are Lagrangian and the Lagrangian/Lagrangian intersections will easily yield the isotropic/coisotropic intersections needed in Corollary 6.2 $\left(W^{u u}\left(a_{k}\right)\right.$ and $W^{s}(N)$ transversely intersecting at $\left.c_{k}\right)$. We start with a reminder on Arnold's example and define the objects $(F$, $M, N$, the transition chain,...) needed to set up the context of Corollary 6.2.

The autonomous version of the Hamiltonian used by Arnold is defined on $\mathbb{T}^{3} \times \mathbb{R}^{3}$ and is given by

$$
H_{\varepsilon, \mu}(\theta, r)=\frac{1}{2}\left(r_{1}^{2}+r_{2}^{2}\right)+r_{3}+\varepsilon\left(\cos \theta_{1}-1\right)+\varepsilon \mu\left(\cos \theta_{1}-1\right)\left(\cos \theta_{2}+\sin \theta_{3}\right),
$$

where $\theta=\left(\theta_{1}, \theta_{2}, \theta_{3}\right) \in \mathbb{T}^{3}, r=\left(r_{1}, r_{2}, r_{3}\right) \in \mathbb{R}^{3}$ and $0<|\mu|<<|\varepsilon|<<1$.

Theorem 6.3. [Arnold] Given $A<B$, there exists $\varepsilon_{0}>0$ such that for all $\left.\varepsilon \in\right] 0 ; \varepsilon_{0}$ [ there exists $\mu_{0}$ such that for all $\left.\mu \in\right] 0 ; \mu_{0}\left[\right.$, the system $H_{\varepsilon, \mu}$ admits an orbit whose projection on the action space $\mathbb{R}^{3}$ intersects the open sets $r_{2}<A$ and $r_{2}>B$.

The Hamiltonian $H_{\varepsilon, \mu}$ is a perturbation of $H_{0,0}=\frac{1}{2}\left(r_{1}^{2}+r_{2}^{2}\right)+r_{3}$, and the parameters $\varepsilon$ and $\mu$ play asymmetric roles: $\varepsilon$ preserves the integrability and creates hyperbolicity, and $\mu$ breaks down the integrability and causes instability. More precisely, when $\varepsilon=0, \mathbb{T}^{3} \times \mathbb{R}^{3}$ is foliated by invariant lagrangian tori, and when $\varepsilon>0$ and $\mu=0$, the system is equivalent to the uncoupled product of a pendulum $\left(H_{p}\left(\theta_{1}, r_{1}\right)=\frac{1}{2} r_{1}^{2}+\varepsilon\left(\cos \theta_{1}-1\right)\right)$ with the completely integrable system $H_{r}\left(\theta_{2}, \theta_{3}, r_{2}, r_{3}\right)=\frac{1}{2} r_{2}^{2}+r_{3}$. The resonant surface given by the equation $r_{1}=0$, which is invariant and foliated by invariant tori when $\varepsilon=0$, is destroyed. It gives rise to a one-parameter family of 2-dimensional invariant tori which are partially hyperbolic, whose union is the normally hyperbolic invariant manifold $N^{\prime}:=\{0,0\} \times \mathbb{T}^{2} \times \mathbb{R}^{2}$. The invariant manifolds of $N^{\prime}$ are the product of those of the hyperbolic point $\left(\theta_{1}=0, r_{1}=0\right)$ with the annulus $\mathbb{T}^{2} \times \mathbb{R}^{2}$. When $|\mu|>0$, we lose the integrability and the invariant manifolds of the tori do not coincide anymore. The Poincaré-Melnikov integrals show that there exists $\varepsilon_{0}>0$ such that for all $\left.\varepsilon \in\right] 0 ; \varepsilon_{0}$ [ there exists $\mu_{0}$ such that for all $\left.\mu \in\right] 0 ; \mu_{0}$ [, the invariant manifolds transversely intersect along a homoclinic orbit. Note that Arnold chose the last term of the perturbation in such a way that it vanishes on the invariant tori (because $\theta_{1}=0$ ), and thus the previous partially hyperbolic tori are preserved when $\mu>0$, as well as the normally hyperbolic manifold. 
It is possible to choose a section $\mathcal{S}$ (see [Mar96]) contained in an energy level $\mathfrak{H}$ and transverse (in $\mathfrak{H}$ ) to the Hamiltonian flow. The Poincaré map associated to $\mathcal{S}$ and defined in a neighborhood of $N:=N^{\prime} \cap \mathcal{S}$ (which is also normally hyperbolic) in $\mathcal{S}$ will play the role of $F$ (this of course is immediate with the nonautonomous form of the system). Note that $\mathcal{S}$ can be chosen so that the invariant manifolds of $N$ are the intersections of those of $N^{\prime}$ with $\mathcal{S}$.

Let $\omega$ be irrational and let $T_{\omega}$ be the torus in $N$ given by the equation $r_{2}=\omega$. It is invariant and minimal (because $\omega$ is irrational). Arnold proved the existence of a finite family $\left(T_{\omega_{i}}\right)_{i \in I}$ of those tori that have in addition Lagrangian invariant manifolds with transverse heteroclinic connections: $W^{u}\left(T_{\omega_{i}}\right) \pitchfork W^{s}\left(T_{\omega_{i+1}}\right)$.

To get Arnold's orbits, it suffices now to apply Corollary 6.2 to the family $\left(T_{\omega_{i}}\right)$, since this family is contained in a normally hyperbolic manifold. The Lagrangian/Lagrangian intersection implies the isotropic/coisotropic intersection needed in the corollary. More precisely, for all $i \in I$, let $c_{i} \in W^{u}\left(T_{\omega_{i}}\right) \pitchfork W^{s}\left(T_{\omega_{i+1}}\right)$. We set $a_{i}$ the point in $T_{\omega_{i}}$ such that $c_{i} \in W^{u u}\left(a_{i}\right)$. It is easy to see that $W^{u u}\left(a_{i}\right)$ transversely intersects $W^{s}(N)$ at $c_{i}$. One gets then a transition chain as in Corollary 6.2, 


\section{References}

[Arn64] V.I. Arnold, Instability of dynamical systems with several degrees of freedom, Sov. Math. Doklady 5 (1964), 581-585.

[BB] P. Berger and A. Bounemoura, A geometrical proof of the persistence of normally hyperbolic submanifolds, Submitted.

[BM11] A. Bounemoura and J.-P. Marco, Improved exponential stability for quasi-convex Hamiltonians, Nonlinearity 24 (2011), no. 1, 97-112.

[Bos86] J.-B. Bost, Tores invariants des systèmes dynamiques Hamiltoniens (d'après Kolmogorov, Arnol'd, Moser, Rüssmann, Zehnder, Herman, Pöschel,...). (Invariant tori of Hamiltonian dynamical systems)., Sémin. Bourbaki, 37e année, Vol. 1984/85, Exp. No.639, Astérisque 133/134, 113-157 (1986)., 1986.

[Cha04] M. Chaperon, Stable manifolds and the perron-irwin method, Ergodic Theory dynam. systems 24 (2004), no. 5, 1359-1394.

[Cre00] J. Cresson, Un $\lambda$-lemme pour des tores partiellement hyperboliques, C. R. Acad. Sci. Paris Sér. I Math. 331 (2000), no. 1, 65-70.

[Cre01] _ Temps d'instabilité des systèmes hamiltoniens initialement hyperboliques, C. R. Acad. Sci. Paris Sér. I Math. 332 (2001), no. 9, 831-834.

[CW] J. Cresson and S. Wiggins, A ג-lemma for normally hyperbolic invariant manifold, unpublished.

[DDLLS06] A. Delshams, R. De La Llave, and T. M. Seara, A geometric mechanism for diffusion in hamiltonian systems overcoming the large gap problem: heuristics and rigorous verification on a model, Memoirs of the American Mathematical Society 179 (2006), no. 844, viii+141 pp.

[DH11] A. Delshams and G. Huguet, A geometric mechanism of diffusion: Rigorous verification in a priori unstable Hamiltonian systems, J. Differential equations 250 (2011), 2601-2623.

[FM00] E. Fontich and P. Martìn, Differentiable invariant manifolds for partially hyperbolic tori and a lambda lemma, Nonlinearity 13 (2000), no. 5, 1561-1593.

[GR07] M. Gidea and C. Robinson, Shadowing orbits for transition chains of invariant tori alternating with Birkhoff zones of instability, Nonlinearity 20 (2007), no. 5, 11151143.

[GR09] _ Obstruction argument for transition chains of tori interspersed with gaps, Discrete Contin. Dyn. Syst. Ser. S 2 (2009), no. 2, 393-416.

[HK95] B. Hasselblatt and A. Katok, Introduction to the modern theory of dynamical systems, Encyclopedia of Mathematics and Its Applications. 54. Cambridge: Cambridge University Press. xviii, 802 p. , 1995.

[HPS77] M.W Hirsch, C.C. Pugh, and M. Shub, Invariant manifolds, Lecture Notes in Mathematics, vol. 583, Springer Verlag, 1977. 
[LM05] P Lochak and J.P. Marco, Diffusion times and stability exponents for nearly integrable analytic systems, Central European Journal of Mathematics 3 (2005), no. 3, 342-397.

[LMS03] P. Lochak, J.-P. Marco, and D. Sauzin, On the splitting of invariant manifolds in multidimensional near-integrable hamiltonian systems, Memoirs of the American Mathematical Society 163 (2003), no. 775.

[Loc99] Pierre Lochak, Arnold diffusion ; a compendium of remarks and questions, Simó, Carles (ed.), Hamiltonian systems with three or more degrees of freedom. Proceedings of the NATO Advanced Study Institute, 1995. Dordrecht: Kluwer Academic Publishers., 1999.

[Mara] J.-P. Marco, - Lecture 9 - Homoclinic and heteroclinic connections, Preprint.

[Marb] - Uniform lower bounds of the splitting for analytic near-integrable systems, Preprint.

[Mar96] _ Transition le long de chaînes de tores invariants pour les systèmes Hamiltoniens analytiques, Ann. Inst. H. Poincaré 64 (1996), no. 2, 205-252.

[MS02] J.-P. Marco and D. Sauzin, Stability and instability for Gevrey quasi-convex nearintegrable Hamiltonian systems, Publ. Math. Inst. Hautes Études Sci. 96 (2002), 199-275.

[MS04] _ Wandering domains and random walks in Gevrey near-integrable systems, Erg. Th. Dyn. Sys. 5 (2004), 1619-1666.

[Wig94] S. Wiggins, Normally hyperbolic invariant manifolds in dynamical systems, Springer Verlag, 1994.

[Zha11] Ke Zhang, Speed of arnold diffusion for analytic hamiltonian systems, Inventiones Mathematicae 186 (2011), 255-290, 10.1007/s00222-011-0319-6. 